\title{
The influence of viral infection on a plankton ecosystem undergoing nutrient enrichment
}

C. J. Rhodes ${ }^{* 1,2}$ and A. P. Martin ${ }^{3}$

1. Institute for Mathematical Sciences, Imperial College London, 53 Prince's Gate, Exhibition Road, South Kensington, London, SW7 2PG, United Kingdom.

2. MRC Centre for Outbreak Analysis and Modelling, Imperial College London, Norfolk Place, London, W2 1PG, United Kingdom.

3. National Oceanography Centre, Southampton, SO14 3ZH, United Kingdom.

\section{c.rhodes@imperial.ac.uk}

tel: $\quad+44(0) 2075941753$

Fax: $\quad+44(0) 2075940923$

*Corresponding author

\begin{abstract}
It is increasingly recognised that viruses are a significant active component of oceanic plankton ecosystems. They play an important role in biogeochemical cycles as well as being implicated in observed patterns of species abundance and diversity. The influence of viral infection in plankton ecosystems is not fully understood. Here we use a number of well-founded mathematical models to investigate the interplay of the ecological and epidemiological interactions of plankton and viruses in the sea. Of particular interest is the role of nutrient on the population dynamics. Nutrient forcing has been suggested as a means of absorbing excess anthropogenic atmospheric carbon dioxide by stimulating increased phytoplankton primary productivity. Here we show that enriching nutrient levels in the sea may decrease the amount of infected phytoplankton thereby additionally enhancing the efficiency of the biological pump, a means by which carbon is transferred from the atmosphere to the deep ocean.
\end{abstract}

Keywords: phytoplankton, predator-prey modelling, zooplankton, viral infection, ecological modelling, nutrient enrichment, climate change, geo-engineering. 


\section{Introduction}

Viruses are the most abundant organism in the world's oceans, and it is thought that all phytoplankton species are susceptible to infection (Bergh et al., 1989; Bratbak et al., 1990; Suttle et al., 1990; Fuhrman, 1999; Evans et al., 2003; Suttle, 2005). Developing an understanding of the role of viruses in oceanic ecosystems is important because the action of viral lysis converts phytoplankton into more viruses and cellular debris, resulting in elevated levels of particulate and dissolved organic matter in the surface regions where the phytoplankton are predominantly found. This process "short-circuits" the transfer of carbon to organisms at higher trophic levels thereby preventing the subsequent transport of carbon (and other inorganic nutrients) to depth as detritus - a process known as the "biological pump". Moreover, the enhanced particulate organic matter in the euphotic zone can be photo-oxidised by sunlight resulting in transfer of carbon from the ocean to the atmosphere (Suttle, 2005).

These processes result in differences in how carbon is exchanged between the sea and the atmosphere when virus is either present or absent, and this has consequences for carbon cycle modelling and climate change estimates (Sabine et al., 2004). From an ecological perspective, phytoplankton are the base species of most oceanic food chains, so they significantly influence the population dynamics and community structure of many higher organisms (Kaiser et al., 2005). Of significant topical interest, oceanic sequestration of excess atmospheric carbon dioxide from anthropogenic sources has been proposed as a means of reducing the rate of global warming (Lampitt et al., 2008). One suggestion is that fertilizing areas of low surface nutrient concentrations will boost phytoplankton growth and increase the flux of carbon to the deep sea (Lampitt et al., 2008; Lovelock and Rapley, 2007). Understanding the responses of plankton ecosystems to exogenous nutrient forcing is, therefore, an essential pre-requisite to implementing large-scale open ocean geoengineering schemes (Chisholm, 2001).

Phytoplankton populations are subject to numerous regulating constraints which, in combination, determine their observed population density at a given time. Whilst viruses are believed to play a significant role in regulating phytoplankton numbers, an additional important constraint on phytoplankton growth is the availability of nutrient. 
This often takes the form of trace quantities of nitrogen (predominantly in nitrate form), though phosphorous and iron concentrations also influence growth (Arrigo, 2005). The abundance of nutrient is variable in both space and time. Nutrient can be brought to the euphotic region, where there is sufficient light to allow photosynthesis, from deeper down by a number of physical processes including upwelling and smallscale mixing. Lateral advection of nutrients into a region may also occur. In particular, rivers can provide a large flux of nutrients near coastal regions. The control of phytoplankton growth by nutrient availability is referred to as 'bottom-up' control. 'Top-down' control, predation by higher organisms (from zooplankton to pelagic fishes), is also a major determinant of phytoplankton numbers.

Therefore, it would appear to be the case that phytoplankton (and zooplankton) abundance is strongly determined by both ecological and epidemiological constraints. Here we use biologically well-founded models of varying complexity to investigate how these two constraints influence the observed plankton population dynamics. Ecological modelling and epidemiological modelling have long and rich histories, whereas the subject of "eco-epidemic" modelling, where the effects of ecology and disease epidemiology mutually interact, is somewhat less developed (Anderson and May, 1986; Chattopadhyay and Arino, 1999; Xiao and Chen, 2001; Chattopadhyay and Pal, 2002; Hudson et al., 2002; Greenhalgh and Haque, 2007, Siekmann et al., 2008). However, enlarging our understanding of the observed patterns of phytoplankton and zooplankton population dynamics provides a strong motivation for investigating the properties of eco-epidemic models. Of particular interest in what follows is the role of nutrient on the various constituent populations in the models. It was Rosenzweig $(1971,1972)$ who, as a consequence of a study on the stability of enriched ecosystems, first drew attention to the possibility that increasing the supply of limiting nutrients to a prey species could lead to a strong increase in predator biomass whilst the prey abundance remained relatively unaffected. Here, we too focus attention on the effects that are caused by changes in the availability of nutrient, though we use combined ecological-epidemic models.

Experience with a single species ecological model with a fatal infectious pathogen, but lacking grazing of phytoplankton by zooplankton (Appendix A), indicates that increasing the nutrient level will also increase the density of infection. We investigate 
three different models of a plankton ecosystem subject to fatal viral infection in the phytoplankton, but with explicit representation of zooplankton and grazing. The sequence of three models is chosen so as to give confidence in the robustness of the particular phenomenon reported here. Specifically we show that in all the models the abundance of infected phytoplankton drops with increasing nutrient level. Moreover, in all the models there is a critical level of nutrient above which the infected phytoplankton (and virus) is completely eliminated from the system. This is a somewhat unexpected result. It suggests there might be a negative correlation between observed ambient nutrient level and viral abundance and that very high levels of nutrient might eliminate virus altogether. The motivation for this paper is to present results that should stimulate more experimental work on this issue. Given the importance of phytoplankton in the oceanic ecosystem and the global carbon cycle, understanding the role of nutrient is important particularly if actions are taken to deliberately manipulate ambient ocean nutrient levels as a means of mitigating anthropogenic global warming (Chisholm, 2000, 2001; Lovelock and Rapley, 2007; Lampitt et al., 2008).

In Section 2 we describe the three models in turn, and in Section 3 we present some supporting evidence from at-sea measurements conducted during a phytoplankton bloom. Conclusions based on the analysis and numerical work are summarised in Section 4. Additional analysis of the models is presented in Appendices.

\section{Eco-epidemic models}

The effects of communicable disease on organisms that are part of a wider ecosystem have been most frequently studied in the context of wildlife diseases. Often these are terrestrial ecosystems involving micro- and macro-parasitic diseases in mammals or invertebrates. Illustrative of this approach are the collected papers in Grenfell and Dobson (1995) or, more recently, Hudson et al. (2002). By contrast, though there is increasing recognition of the role played by viruses in the sea, representative models of virally infected plankton ecosystems have been less fully elaborated. Studies of the 
effect of phytoplankton virus in planktonic ecologies include those of Beltrami and Carroll (1994), Chattopadhyay and Pal (2002), Chattopadhyay et al. (2003), Thyraug et al. (2003), Singh et al. (2004), Malchow et al. (2004), Malchow et al. (2005), Hilker and Malchow (2006) and Siekmann et al. (2008). More recently Rhodes, Truscott and Martin (2008) extended the ecological models of Truscott and Brindley (1994), Oschlies and Garçon (1999) and Martin et al. (2002) to investigate the effect of virus infection in transient plankton blooms and pelagic plankton.

In what follows we extend our recent work to study the effect of eutrophication of plankton ecosystems using density dependent disease transmission terms in a virally infected phytoplankton population that is also subject to predation. Specifically, we assume a lytic viral transmission mechanism. We do so for two reasons. First, there is evidence that it is more common in marine phytoplankton than lysogenic transmission (Fuhrman, 1999). Second, lysis of cells releases their component carbon, nitrogen, iron and phosphorous back into the ambient pool. This potentially has a major effect on global biogeochemical cycles as it means that the transport of carbon to depth away from the atmosphere via sinking organic material is reduced.

The models of Beltrami and Carroll (1994), Malchow et al. (2004), Malchow et al. (2005) and Hilker and Malchow (2006) use a frequency dependent transmission term (and sometimes assume lysogenis). There is currently insufficient empirical evidence to determine which of frequency or density dependent transmission is most appropriate in a model. Therefore, we invoke Occam's razor and have chosen density dependent transmission, where the rate of acquisition of new infection is proportional to the product of the densities of infected and uninfected phytoplankton, as this requires fewer assumptions about the dynamics of transmission.

Predation of the phytoplankton is a significant determinant of observed population densities. We assume that zooplankton is unable to discriminate the disease status of the phytoplankton, and so they predate the infected and uninfected phytoplankton to the same degree. By contrast, in Chattopadhyay and Pal (2002) only the uninfected prey are predated, and in Chattopadhyay et al. (2003) there is no predation of phytoplankton by organisms at higher trophic levels. 
A further key assumption concerns the growth of phytoplankton. We assume that infected phytoplankton do not influence the growth rate of uninfected phytoplankton. Following viral infection it is assumed that the infected phytoplankton simply act as sources of fresh virus and as food for zooplankton. This differs from the assumption made by Singh et al. (2004) where the infected phytoplankton does influence the growth rate of uninfected phytoplankton. Furthermore, we specify that infected phytoplankton do not reproduce and that phytoplankton population growth comes only from uninfected organisms.

The coupling of ecological models with epidemiological ones can lead to quite large and complex models with many associated parameters. This can make it challenging to determine whether observed behaviours are robust features of such systems or rather the result of some particular combination of the chosen functional parameterisations (of predation or infection, for example) and model parameters. Recalling that our focus here is on the effects caused by nutrient enrichment we analyse a sequence of increasingly elaborate eco-epidemic models. The first model uses a minimal representation of the ecological-epidemiological interaction in a plankton-virus system. The subsequent models use more complex predation functions and a different zooplankton mortality function. There are currently insufficient data to constrain species-specific models, so the models presented here represent "generic" species. In reality there will be greater complexity, including perhaps inter-specific competition between multiple phytoplankton species that are themselves subject to predation and viral attack, and possibly multiple virus strains. The strategy adopted here of using several models with differing structural forms is intended to reinforce confidence in any conclusions that are drawn.

\subsection{Model 1}

In the absence of predation or infection we assume that the phytoplankton population growth is a logistic process with a growth rate, $a$, and a carrying-capacity, $K$. When viral infection is present the phytoplankton population will be partitioned into "susceptible" (i.e. uninfected) and "infected" classes. The simplest representations of 
predation and infection are bilinear forms. A set of dynamical equations for the evolution of the susceptible phytoplankton, $P_{s}$, the infected phytoplankton, $P_{i}$, and the zooplankton, $Z$, is given by:

$$
\begin{gathered}
\frac{d P_{s}}{d t}=a P_{s}\left(1-\frac{P_{s}}{K}\right)-R_{m} Z P_{s}-\beta P_{s} P_{i} \\
\frac{d P_{i}}{d t}=\beta P_{s} P_{i}-R_{m} Z P_{i}-\alpha P_{i} \\
\frac{d Z}{d t}=\delta R_{m} Z\left(P_{s}+P_{i}\right)-\mu Z
\end{gathered}
$$

It is assumed that only the uninfected phytoplankton reproduce and increase in number but that both infected and susceptible phytoplankton are predated with equal vigour by the zooplankton, and that conversion of susceptible and infected phytoplankton biomass to zooplankton biomass is commensurable (as zooplankton recognise no difference between infected and uninfected phytoplankton) but is not $100 \%$ efficient, occurring at a level determined by $\delta$. Infected phytoplankton does not reproduce. Zooplankton mortality is assumed linear in density $Z$, at a rate $\mu$. The viral transmission mechanism is taken to be a density dependent form with a transmission parameter $\beta$, and infected phytoplankton is assumed to die at a rate set by $\alpha$. In order to keep the model simple it is assumed that the viruses that are shed by the lysis of infected phytoplankton are in equilibrium with the creation of infected phytoplankton. Consequently, the rate of acquisition of newly infected cells is proportional to the density of infected phytoplankton thereby obviating the need for a further equation describing the time evolution of the free virus population. Model 1 is structurally similar to one of the variants of equation 27 first presented in Anderson and May (1986). We use a logistic growth term for the prey population. Furthermore, we enforce identical predation of infected and susceptible prey. 


\subsubsection{Analysis of Model 1 - Ecosystem component}

Assuming that there is no virus present, or that the transmissibility is too low to sustain endemic infection, the model reverts to a basic predator-prey model for the phytoplankton-zooplankton interaction. In this case the non-trivial equilibrium values of the phytoplankton and zooplankton populations are

$P^{*}=\mu / \delta R_{m}$ and $Z^{*}=\frac{a}{R_{m}}\left(1-\frac{\mu}{K \delta R_{m}}\right)$. It can be seen that as $K$ becomes larger (a proxy for the increase of nutrient input), so the zooplankton population density is increased whereas the phytoplankton population remains unaffected. This is an observation originally made in the context of nutrient enriched ecosystems by Rosenzweig (1971). However, as nutrient input becomes large $(K \rightarrow \infty)$ the steady state populations remain stable, so strong enrichment of the ecosystem component does not lead to destabilisation. Appendix B outlines a stability analysis of this model.

\subsubsection{Analysis of Model 1 - Full Model}

If we now permit a finite $\beta$ it becomes possible for virus to exist in the ecoepidemiological system. First, we find the non-trivial equilibrium solutions of equations $1-3$. This gives

$$
\begin{gathered}
P_{s}^{*}=K-\frac{K}{a}\left(\frac{\beta \mu}{\delta R_{m}}-\alpha\right) \\
P_{i}^{*}=\left(\frac{\mu}{\delta R_{m}}-K\right)+\frac{K}{a}\left(\frac{\beta \mu}{\delta R_{m}}-\alpha\right) \\
Z^{*}=\frac{\beta K}{R_{m}}\left(1-\frac{\beta \mu}{\delta R_{m} a}+\frac{\alpha}{a}\right)-\frac{\alpha}{R_{m}}
\end{gathered}
$$


Note that the total phytoplankton biomass $P_{s}^{*}+P_{i}^{*}=\mu / \delta R_{m}$, which is independent of nutrient input, $K$, or viral transmissibility, $\beta$. Hence, instead of discussing absolute abundance for this model, we can instead focus unambiguously on prevalence, the total fraction of phytoplankton that is infected. (This is also true for Model 2 but the variation of total phytoplankton abundance with $K$ for Model 3 precludes this.) If we insist on $P_{i}^{*}>0$ (a finite density of infected phytoplankton at an equilibrium level, i.e. stable endemic infection) (5) can be used to show that for this to happen

$$
\beta>\frac{\delta R_{m}}{\mu}(\alpha+a)-\frac{a}{K}
$$

This gives a critical value for the virus transmission parameter above which there will be persistent viral infection and below which the virus cannot exist indefinitely in phytoplankton. What is apparent from equation 7 is that as the carrying capacity $K$ increases (a proxy for rising nutrient input), so it becomes necessary for the virus to be increasingly transmissible (larger $\beta$ ) in order for it to maintain its presence at a given level in the ecosystem. This suggests that enrichment of the oceanic ecosystem as represented by this model reduces the prevalence of viral infection in the phytoplankton population, whilst having no effect on overall phytoplankton $\operatorname{biomass}\left(P_{s}+P_{i}\right)$.

By differentiating equation 5 it is possible to see how the prevalence of viral infection in the phytoplankton depends on $K$. If

$$
\left(\frac{\beta \mu}{a \delta R_{m}}-\frac{\alpha}{a}\right)<1
$$

then $\partial P_{i}^{*} / \partial K<0$ corresponding to a decreasing density of infected phytoplankton with increasing $K$. The inequalities in equations 7 and 8 when taken together specify the extent of the interval in which the viral transmissibility $\beta$ must sit in order for viral prevalence to decrease with increasing nutrient, namely 


$$
\frac{\delta R_{m}}{\mu}(a+\alpha)-\frac{a}{K}<\beta<\frac{\delta R_{m}}{\mu}(a+\alpha)
$$

As nutrient enrichment progresses ( $K$ increases) the interval of transmissibility over which $\partial P_{i}^{*} / \partial K<0$ (of width $a / K$ ) gets narrower.

However, the upper threshold is an over-estimate as a bifurcation at a lower value of $\beta$ $\left(\beta_{c}\right)$ leads to loss of the stable steady state with a non-zero zooplankton population, and the ecosystem collapses to a purely epidemiological one, consisting of uninfected and infected phytoplankton and virus only. The details of this are presented in Appendix C, and it suggests that a transition from a state $\left(P_{s}^{*}, P_{i}^{*}, Z^{*}\right) \rightarrow\left(P_{s}^{*}, P_{i}^{*}, 0\right)$ occurs before the upper critical threshold of transmissibility is passed.

Figure 1 shows of the behaviour of the basic eco-epidemic model as a function of viral transmissibility $\beta$. For values of $\beta$ that are too small to support endemic viral infection, the system is purely ecological with phytoplankton and zooplankton coexisting in stable equilibrium. This occurs to the left of the left-hand vertical dashed line and in this region there is no change in phytoplankton density as nutrient is altered, i.e. $\partial P / \partial K=0$. Here $P$ denotes the total phytoplankton density, because $P_{i}=0$. As $\beta$ increases a threshold to endemic viral infection, above which virus can persist in the planktonic ecosystem, is crossed, and the prevalence of infected phytoplankton increases with increasing transmissibility as expected. However, if nutrient enrichment occurs in this region there will be a resulting decline in the prevalence of infected phytoplankton. The region where this occurs is represented by the interval to the right of the left-hand vertical dashed line up to the right hand dashed line. For sufficiently high transmissibility $\left(\beta>\beta_{c}\right)$ zooplankton is eliminated and the system becomes an epidemiological one consisting of uninfected and infected phytoplankton only. This occurs to the right of the vertical solid line. In this region, any enrichment of the system results in increasing prevalence of infected 
phytoplankton (Appendix C, equation C5). The vertical dashed line indicates the upper limit in Equation 9.

Consequently, there may be a declining prevalence of virally infected phytoplankton in nutrient enriched planktonic-viral ecosystems when zooplankton is present. The right hand vertical dashed line is the upper limit in equation 9. From equation $\mathrm{C} 4$, the width of the interval between zooplankton being extinguished, $\beta_{c}$, and this line is $\alpha /[K(1+\alpha / a)]$. The width of the interval of $\beta$ that permits $\partial P_{i}^{*} / \partial K<0$ is therefore $\mathrm{a} / K-\alpha /[K(1+\alpha / \mathrm{a})]$. It is only possible to increase the proportion of total phytoplankton that is infected by nutrient enrichment when zooplankton has been eliminated from the ecosystem, i.e. in the region to the right of $\beta_{c}$. This situation generally does not arise in the ocean where zooplankton, phytoplankton and virus co-exist.

From equation 5 it is possible to derive a critical value of $K\left(K_{c}\right)$ above which the infectious phytoplankton will be eliminated. Choosing a viral transmissibility that enables virus to co-exist with phytoplankton and zooplankton, a progressive increase in nutrient decreases the prevalence of infected phytoplankton, until at some critical value of nutrient, they are eliminated altogether leaving only a phytoplankton and zooplankton ecosystem. The critical value of nutrient at which this happens is given by

$$
K_{c}=\frac{\mu / \delta R_{m}}{\left(1-\frac{\beta \mu}{a \delta R_{m}}+\frac{\alpha}{a}\right)}
$$

Figure 2a shows how the eco-epidemiological system behaves as the nutrient level is increased. For increasing $K\left(<K_{c}\right)$ the proportion of infected phytoplankton declines until it is eliminated for nutrient levels $>K_{c}$. The dots show the zooplankton biomass increases with nutrient level, whereas the overall phytoplankton density remains constant. Figure $2 \mathrm{~b}$ indicates how the density of infected phytoplankton varies with $K$ and $\beta$. The contour lines clearly show the decline in infection as $K$ increases. 


\subsubsection{Summary of Model 1}

From the analysis of this basic eco-epidemic model it is apparent that the ecosystem component of the model conforms to Rosenzweig's result $(1971,1972)$ that additional nutrient will get sequestrated in the predator population (in this case zooplankton) rather than in the prey population (here the phytoplankton). It is noted that this effect is maintained in the presence of viral infection in the prey population. Furthermore, when viral infection is introduced into the planktonic ecosystem there is a critical threshold value of transmissibility above which the virus can persist indefinitely in the phytoplankton population and below which it will go to extinction. As the nutrient level is increased the prevalence of a virus of a given transmissibility will decline until, for sufficiently large $K\left(K_{c}\right)$ it will be eliminated. Note that the total density of phytoplankton $\left(P_{s}+P_{i}\right)$ is unaffected by the nutrient level and is independent of the value of transmissibility $\beta$; rather, it is the relative proportion of uninfected and infected phytoplankton that is affected by the nutrient level. Therefore, nutrient enrichment has the perhaps surprising result of decreasing the prevalence of infected phytoplankton relative to the uninfected phytoplankton. (Enrichment of a nonpredated infected species undergoing logistic growth leads to increased prevalence of infected prey - Appendix A).

\subsection{Model 2}

In order to test the generality of the conclusions we have reached using Model 1, we now introduce a more sophisticated functional form for the predation of the phytoplankton by zooplankton. In practice it takes time for a zooplankton to identify, capture and consume its phytoplankton prey. Following Holling, this leads to a saturating limit for the rate of prey consumption (Holling, 1959, Begon et al., 2005). Using a Holling Type II functional form we now have 


$$
\begin{gathered}
\frac{d P_{s}}{d t}=a P_{s}\left(1-\frac{P_{s}}{K}\right)-\frac{\lambda Z P_{s}}{g+\left(P_{s}+P_{i}\right)}-\beta P_{s} P_{i} \\
\frac{d P_{i}}{d t}=\beta P_{s} P_{i}-\frac{\lambda Z P_{i}}{g+\left(P_{s}+P_{i}\right)}-\alpha P_{i} \\
\frac{d Z}{d t}=\frac{\delta \lambda Z\left(P_{s}+P_{i}\right)}{g+\left(P_{s}+P_{i}\right)}-\mu Z
\end{gathered}
$$

All parameters are as before, though now $g$ is the half-saturation constant for grazing and $\lambda$ is the maximum zooplankton grazing rate. This model is an extension of Model 1 to include Type II predation with all other biological assumptions remaining the same.

The objective here is to determine whether the characteristic behaviour that relates to Model 1 also appear in this model with its more refined representation of predation.

\subsubsection{Analysis of Model 2 - Ecosystem Component}

In the absence of virus the system is a purely ecological one consisting of zooplankton and their prey phytoplankton. The stable fixed point of these populations is at

$$
P^{*}=\frac{\mu g}{\delta \lambda-\mu}
$$

And

$$
Z^{*}=\frac{1}{\lambda}\left(g+\frac{\mu g}{\delta \lambda-\mu}\right)\left(a-\frac{a}{K} \frac{\mu g}{\delta \lambda-\mu}\right)
$$

As in Model 1, following Rosenzweig (1971), if $K$ increases as nutrient input is enhanced, then the equilibrium biomass of zooplankton is increased, whereas the 
phytoplankton remains unaffected. Appendix D outlines a stability analysis of this solution.

\subsubsection{Analysis of Model 2 - Full Model}

The non-trivial equilibrium solutions of equations $11-13$ are as follows:

$$
\begin{gathered}
P_{s}^{*}=K-\frac{K}{a}\left(\frac{\beta \mu g}{\delta \lambda-\mu}-\alpha\right) \\
P_{i}^{*}=\left(\frac{\mu g}{\delta \lambda-\mu}-K\right)+\frac{K}{a}\left(\frac{\beta \mu g}{\delta \lambda-\mu}-\alpha\right) \\
Z^{*}=\left(g+\frac{\mu g}{\delta \lambda-\mu}\right)\left(\frac{\beta P_{s}^{*}-\alpha}{\lambda}\right)
\end{gathered}
$$

assuming $(\delta \lambda-\mu)>0$. Note that $P_{s}^{*}+P_{i}^{*}=\mu g /(\delta \lambda-\mu)$ implying that the overall density of phytoplankton is independent of nutrient level and viral transmissibility. For virus to be endemic in the system we require $P_{i}^{*}>0$. For this to be true, the critical viral transmissibility must be

$$
\beta>\frac{(\delta \lambda-\mu)}{\mu g}(a+\alpha)-\frac{a}{K}
$$

As above, for $(\beta \mu g / a(\delta \lambda-1)-\alpha / a)<1$ we find that $\partial P_{i}^{*} / \partial K<0$. So, the constraint on the viral transmissibility (equivalent to equation 9) is

$$
\frac{(\delta \lambda-\mu)}{\mu g}(a+\alpha)-\frac{a}{K}<\beta<\frac{(\delta \lambda-\mu)}{\mu g}(a+\alpha)
$$


As nutrient enrichment progresses ( $K$ increases) the interval of transmissibility over which $\partial P_{i}^{*} / \partial K<0$ (of width $a / K$ ) gets narrower, in the same way as Model 1.

As $\beta$ passes through the upper threshold, $\beta \sim(\delta \lambda-\mu)(\mathrm{a}+\alpha) /(\mu \mathrm{g})$, (getting larger) the zooplankton population is eliminated and there is a transition to a purely epidemiological model consisting of only infected and uninfected phytoplankton (Appendix E). Consequently, it is only possible to observe $\partial P_{i}^{*} / \partial K<0$ in the model when all populations $\left(P_{s}, P_{i}, Z\right)$ are co-existent. The width of the interval of $\beta$ over which $\partial P_{i}^{*} / \partial K<0$ is given by $a / K-\alpha / K(1+\alpha / a)$, as for Model 1 .

Figure 3 shows the behaviour of Model 2 as a function of virus transmissibility $\beta$. As we found in Model 1 above, declining prevalence of infected phytoplankton is the observed behaviour in nutrient enriched ecosystems when zooplankton is present. The vertical dashed line indicates the upper limit in Equation 18.

From equation 15 it is possible to derive a critical value of $K_{c}$ above which the infectious phytoplankton will be eliminated. The critical value of nutrient input at which this happens in Model 2 is given by

$$
K_{c}=\frac{\mu g / \delta \lambda-\mu}{\left(1-\frac{\beta \mu g}{a(\delta \lambda-\mu)}+\frac{\alpha}{a}\right)}
$$

Figure 4a shows how the eco-epidemiological system represented by Model 2 behaves as the nutrient level is increased. Figure $4 \mathrm{~b}$ shows how the density of infected phytoplankton varies with $K$ and $\beta$. 


\subsubsection{Summary of Model 2}

Model 2 was introduced to investigate the effect of a more realistic saturating predation function for the zooplankton and phytoplankton interaction. In most respects the behaviour of Model 2 is very similar to the behaviour of Model 1. In the reduced ecosystem-only version of the model, when virus is stably co-existent with zooplankton and phytoplankton, increasing the nutrient level suppresses the prevalence of infected phytoplankton, as described in relation to Model 1. It should be noted that the conclusions arising from Models 1 and 2 are dependent on the specific choice of trophic closure that is made in equations 3 and 13. This point has been highlighted in other plankton modelling studies (Steele and Henderson 1992; Edwards and Brindley 1999), as the choice of trophic closure is essentially a statement of the assumed ecological mechanism governing zooplankton mortality. For simplicity, equations 3 and 13 use a linear term. An alternative that has been used by other researchers is a quadratic term. In this case if $K\left(\right.$ or $a$ ) is increased, $P_{i}$ increases for small values of $K$ but then saturates so the effect of decreasing $P_{i}$ is absent. Of the use of the linear closure term Steel and Henderson (1992) have stated "There can be a strong empirical basis for this approach if nothing is known (or can be known) about the actual ecological context". Alternatively, it could be argued that a more general closure term might be given by $\mu Z^{2} /(C+Z)$ where $C$ is a constant and we make the assumption that predators of the zooplankton are proportional to them in abundance. Consequently, at low concentrations of zooplankton the closure will be quadratic in $Z$, whereas at higher $Z$ (i.e. at higher nutrient levels), the mortality becomes linear in $Z$. This functional form is more defensible than a simple quadratic as the latter allows an indefinitely increasing specific grazing rate with increasing $\mathrm{Z}$. The former has an upper limit for the specific rate, as would be expected given the physiological constraints on the rate at which a predator can catch, handle and consume prey. Our results are appropriate to the higher $Z$ limit of this more realistic functional form and therefore applicable to the case of increasing nutrient input.

Model 2 is also capable of exhibiting limit cycle behaviour for different parameter choices. When this occurs, eutrophication of the phytoplankton causes an increase in the mean density of infected prey. However, there is no strong evidence that in situ 
oceanic plankton populations are intrinsically oscillatory. Cyclic behaviours are a feature of phytoplankton population dynamics but these are generally attributed to known seasonal effects rather than intrinsic dynamics. Consequently, we do not focus attention on this phase of model behaviour.

\subsection{Model 3}

Although simple models are very good for testing hypotheses care should always be exercised to ensure that any phenomena observed are not model dependent. For this reason we now repeat our analysis using a more sophisticated representation of phytoplankton ecology. Recently, a model was introduced to investigate the role of viral infection in regulating a plankton ecosystem over a seasonal cycle in the North Atlantic (Rhodes et al. 2008). The model, hereafter Model 3, explicitly includes a nutrient state variable that represents the concentration of dissolved nitrate. Explicit state variables for the amount of free virus, V, and detritus, D, are also represented. The only change made to the model relative to that used in Rhodes et al. (2008) is that the zooplankton mortality term is replaced with a linear formulation (rather than a quadratic one) for the argument given in Section 2.2.3.

The model is given by the following equations

$$
\begin{gathered}
\frac{d N}{d t}=s\left(N_{0}-N\right)-\lambda_{\max }\left(\frac{N}{k_{N}+N}\right) P_{s}+\mu_{D} D+\gamma_{2} Z \\
\frac{d P_{s}}{d t}=\lambda_{\max }\left(\frac{N}{k_{N}+N}\right) P_{s}-\left(\frac{g \varepsilon P_{s}\left(P_{s}+P_{i}\right)}{g+\varepsilon\left(P_{s}+P_{i}\right)^{2}}\right) Z-\mu_{P_{s}} P_{s}-\beta P_{s} V \\
\frac{d P_{i}}{d t}=\beta P_{s} V-\left(\frac{g \varepsilon P_{i}\left(P_{s}+P_{i}\right)}{g+\varepsilon\left(P_{s}+P_{i}\right)^{2}}\right) Z-\mu_{P_{i}} P_{i}
\end{gathered}
$$




$$
\begin{gathered}
\frac{d V}{d t}=\gamma P_{i}-\mu_{V} V \\
\frac{d Z}{d t}=\gamma_{1}\left(\frac{g \varepsilon\left(P_{S}+P_{I}\right)^{2}}{g+\varepsilon\left(P_{S}+P_{I}\right)^{2}}\right) Z-\gamma_{2} Z-\mu_{Z} Z \\
\frac{d D}{d t}=\left(1-\gamma_{1}\right)\left(\frac{g \varepsilon\left(P_{s}+P_{i}\right)^{2}}{g+\varepsilon\left(P_{s}+P_{i}\right)^{2}}\right) Z+\mu_{P_{s}} P_{s}+\mu_{P_{i}} P_{i}+\mu_{Z} Z-\mu_{D} D-\frac{w_{s} D}{h}
\end{gathered}
$$

The variables in this model are nutrient (nitrate) $N$, uninfected phytoplankton $P_{s}$, infected phytoplankton $P_{i}$, virus $V$, zooplankton, $Z$ and detrital material $D$. The nitrate pool is partly replenished by re-cycling of dead and excreted material that originates in the zooplankton and phytoplankton. It is possible that decaying material of viral origin could also serve to increase nutrient levels but we have not included that possibility here. Note that, in order to be consistent with Models 1 and 2, we do not allow $P_{i}$ to compete with $P_{s}$ for nutrients. The assumption underlying this is that once phytoplankton become infected they do not reproduce and they do not grow in size. In summary, we assume that nutrient uptake ceases once a cell becomes infected. We revisit this assumption in the Discussion. We further assume that all nitrogen contained in an infected cell becomes part of a virus on lysis so that $\gamma=\mu_{\mathrm{Pi}}$. Other model parameters are as used in Rhodes et al. (2008) to describe the North Atlantic (all parameters held constant throughout the paper are given in Table 1) except for $\beta$ which is discussed below, and $\mu_{Z}$ for which a value of 0.15 day $^{-1}$ was used. Note that although the underlying ecological model has been well-used and studied previously, investigations into plankton viruses are still in their early stages. Consequently, the viral parameters used may vary due to uncertainties in observations and also between species studied. For this reason critical thresholds, of infectivity and nutrient supply, should be treated more qualitatively than quantitatively. The phenomenon is the main focus for now, not the precise parameter values for which it occurs. Model 3 is a more 
complex eco-epidemiological model than has been considered heretofore, so the remaining analysis uses numerical solution of equations 20-25.

As in Models 1 and 2, a virus must be of sufficiently high transmissibility in order to persist in stable equilibrium with the plankton populations. When this is the case, it is possible to investigate the effect that changing nutrient input has on the equilibrium populations of plankton. Using the parameters in Table 1 it turns out that the critical viral transmissibility for stable co-existent persistence $\beta_{\text {crit }} \sim 3.9\left(\mathrm{mmol} \mathrm{N} \mathrm{m}^{-3}\right)^{-1} \mathrm{day}^{-1}$. Therefore, choosing $\beta=4.3\left(\mathrm{mmol} \mathrm{N} \mathrm{m}^{-3}\right)^{-1}$ day $^{-1}$ it is possible to investigate the effect of nutrient input on the eco-epidemiological system. Note that $\beta$ in Model 3 is not directly comparable quantitatively to $\beta$ in Models 1 and 2 because of the explicit representation of the viral population in Model 3. (It must be stressed that other parameter choices are possible for Model 3 but we use those in Table 1 to maintain consistency with the analysis of Rhodes et al. 2008. We do not seek to do an exhaustive search of parameter space. Rather we seek to demonstrate the existence of a phenomenon, robust across a range of models). In this model we use $N_{0}$ to set the rate of nutrient input. (We could alternatively use $s$, and, when doing this, similar results are obtained). The first term on the right hand side of equation 20 can be interpreted as maintaining nitrate input by relaxing its concentration to a value $N_{O}$. Hence, by increasing $N_{0}$ we increase the nitrate input to the system.

Figure 5a shows the response of the equilibrium levels of uninfected and infected phytoplankton to increasing nutrient input, as set by $N_{0}$. Although nutrient enrichment starting from low levels does lead to an increase in the total phytoplankton biomass, the increase in zooplankton abundance is much larger and more rapid. At low nutrient levels, enrichment leads to a slight enhancement in the density of infected phytoplankton, but as enrichment continues to increase, there is a levelling-off followed by a decline in the density of infected phytoplankton. As was seen in the previous two models, there is a critical level of nutrient above which there is complete elimination of the virus and infection from the phytoplankton population. Also, as nutrient level increases the total phytoplankton concentration saturates remaining constant at higher nutrient inputs, similar to Models 1 and 2. 
Figure $5 \mathrm{~b}$ shows the proportions of infected and uninfected in the total phytoplankton population for a given nutrient level. For low nutrient levels the proportion of infected phytoplankton increases slightly relative to the uninfected phytoplankton, whereas for higher nutrient enrichment the proportion of the total phytoplankton that are infected declines.

To explore the behaviour of Model 3 further, it is of interest to investigate the effect that increasing viral transmissibility has on the response to nutrient enrichment. Figure 6 (a-d) shows equivalent calculations to that in Figure 5a, though now for larger values of $\beta$. For increasingly transmissible virus the qualitative behaviour of the infected phytoplankton is preserved, i.e. at low nutrient levels eutrophication increases the density of infected phytoplankton, but for high levels the density begins to decrease, ultimately, leading to elimination. The stable fixed point bifurcates to limit cycle behaviour $(\beta \sim 6.5)$ and represents a highly virulent virus. At typical levels of nutrient (extreme left hand side of Figure 6d) the infected phytoplankton density is around $50 \%$ of the total phytoplankton population, which is rather higher than would be observed in practice. The reason for this behaviour is apparent from Model 1 equation 10; for sufficiently large transmissibility $(\beta)$ in that model the threshold value of $K$ becomes ever larger.

\subsection{Summary of Model 3}

Model 3 was introduced as a more sophisticated model of plankton dynamics in an open ocean setting. In contrast to Model 1 and 2, at low nutrient input levels it is possible to see the density of infectious phytoplankton increasing with increasing nutrient level over a small range. However, further enhancement of nutrient input inevitably leads to a decline in the density of infected phytoplankton, both in absolute terms and as a proportion of the total phytoplankton biomass. Moreover, there is once again a critical nutrient level above which infected phytoplankton is completely eliminated from the system. These are robust features of the behaviour of Model 3. They are preserved when there is a change of trophic closure from $\mu_{Z} Z \rightarrow \mu_{Z} Z^{2}$ and 
also when the re-cycling of detritus and zooplankton excreta is

$\operatorname{excluded}\left(\mu_{D}=0\right.$ and $\left.\gamma_{2}=0\right)$.

Although it may not be readily apparent, Model 3 is not as different from Models 1 and 2 as it appears, so the insights gained from Models 1 and 2 are applicable in the more complex model. It is possible to remove virus from Model 3 by replacing $\mathrm{V}$ with a term proportional to $\mathrm{P}_{i}$ from the steady state solution to equation 23. Furthermore, as we state above, it is possible to turn off recycling of detritus and zooplankton waste products without affecting the phenomenon. Hence the sensitivity analyses we discuss also effectively remove $D$ from the model. This leaves $N, P_{s}, P_{i}$ and $Z$. As we consider nutrient enrichment, nutrients will generally be non-limiting so $N$ plays virtually no role in the dynamics and can also be ignored. Finally by comparing, term-by-term, Model 3 with $V, D$ and $N$ removed with Model 1 or 2 it will be seen that the only differences are: the linear mortality of $P_{s}$ in Model 3 compared to the effective quadratic loss terms in Models 1 and 2; the Holling III grazing term in Model 3 compared to Holling I and II in models 1 and 2 respectively. Hence, it is only the choice of these two functional forms that effectively separates Model 3 from Models 1 and 2.

\section{Experimental Observations}

The analysis of our eco-epidemiological models leads to two predictions that could be tested: i) in the case of single strains of virus the prevalence of the strain will decrease in its host phytoplankton species as sustained nutrient input increases, and ii) in the case of multi-strain viruses, viral diversity will decline with increasing nutrient as less transmissible strains are sequentially eliminated.

Given the counter-intuitive nature of the results shown by all the three models, it is important that attempts are first made to verify the prediction that increased nutrient input can reduce viral lysis. Only limited observations are currently available and none relate exactly to the equilibrium systems we investigate. Figure 7 shows observations taken in situ over the course of two months in the North Sea as the 
ecosystem shifted from a state of high nitrate concentrations to one of low values. This experiment was configured to quantify the contributions to phytoplankton mortality from grazing and viral lysis. What is shown in Figure 7 is the phytoplankton mortality resulting from viral infection (i.e. the virally-induced mortality). The higher the virally-induced mortality the greater the proportion of phytoplankton that are dying due to infection. The corresponding shift from low to high virally-induced mortality is consistent with our modelling results. In the models described above, as nutrient levels fall a given phytoplankton is more likely to die as a result of viral infection. Unfortunately the time series is not long enough to gauge the longer term behaviour of the viral lysis rates which may be more representative of behaviour at equilibrium. Several other studies have traced phytoplankton and viral abundance across the transient responses of spring blooms or in mesoscosms subjected to nutrient 'spikes' (Brussard et al. 2005; Bratbak et al., 1993). Similar behaviour to that shown in Figure 7 has been observed though in some cases, in mesocosm studies, viral attack increased during an induced phytoplankton bloom even when nutrients remained nonlimiting (Bratbak et al. 1993). It will be difficult observationally to separate the phenomenon reported here from the expected increase in viral infection due to burgeoning populations decreasing the distance between potential hosts, particularly during transient events.

\section{Conclusions}

It is becoming increasingly recognised that the abundant and ubiquitous virus populations in the world's oceans are playing a significant role in regulating plankton dynamics and, by implication, the dynamics of populations of species at higher trophic levels. Given the paucity of knowledge relating to phytoplankton epidemiology, much can be learnt from studying basic models that capture the most significant biological processes. In particular, models allow us to develop and frame hypotheses to direct future field and laboratory work.

Here we have investigated the role of nutrient supply as a determinant of phytoplankton dynamics using a sequence of increasingly complex models. This is motivated in part by the suggestion that nutrient enrichment of the seas could serve to 
mitigate the effects of anthropogenic carbon emissions (for a review see Lampitt $e t$ $a l ., 2008)$. Despite their differences, the models have common features. Specifically, there is a pronounced tendency to decreasing phytoplankton infection as nutrient is increased.

It is possible to construct a simple hypothesis that might explain this phenomenon. There are two asymmetries in the model. First, although both susceptible and infected phytoplankton are grazed equally by zooplankton, only susceptible phytoplankton populations increase directly when $K$ is increased. (This is also true if one increases the maximum phytoplankton growth rate a, leaving $K$ constant - see equation 5 ). This asymmetry can only give rise to the observed phenomenon if the increased flux of material into $P_{s}$ is preferentially diverted to $Z$ rather than to $P_{i}$ - otherwise $P_{i}$ may still increase in population size. Second, zooplankton benefit from the increased influx of material whether it is from ingestion of susceptible or infected phytoplankton, but infected phytoplankton only benefit from the fraction of susceptible phytoplankton they can win in competition with zooplankton and further suffer increased losses as a result of increased zooplankton grazing if they lose that competition.

The work reported here assumes that infected phytoplankton do not take up nutrients. At present it is unclear to what extent infected phytoplankton can do. There is some experimental evidence that they may be able to do so and potentially can even increase cellular uptake (Mann et al., 2003). We have carried out further sensitivity analyses to explore this: if Model 3 is modified to allow $P_{i}$ to remove nutrient from the pool but not to use it to reproduce then the phenomenon of increased nutrient loading leading to viral extinction remains (if this is tried with Models 1 or 2 there are no stable equilibrium solutions); at the extreme of allowing infected phytoplankton to uptake nutrient and reproduce identically to uninfected phytoplankton then the phenomenon is not present. It should be remembered that lytic transmission means that no infected cell will reproduce, however. It is not clear how the most realistic scenario of continued uptake of nutrient purely for creation of viruses within an infected cell can be convincingly modelled in the continuous framework used here. Such work may provide an interesting study for individual-based modelling. 
The work presented here focuses on steady state populations. In situ, populations will experience periods of considerable change in nutrient input both on seasonal timescales and shorter ones associated with more ephemeral localised processes. Clearly future work must investigate if the results presented here are modified when the nutrient forcing varies with time. Provided that the system equilibrates as fast as the forcing changes, our results should still be applicable. It is generally thought that increased population density of organisms leads to increased infection at the termination of blooms. Given that the end of a bloom is often coincident with a reduction to low nutrient concentrations, our results suggest that there may be an additional contributory process.

The phenomenon of increased nutrient input leading to reduced viral infection of phytoplankton might have desirable consequences from a human perspective. The reduction or removal of infection would mean that carbon in the phytoplankton is more likely to be consumed by higher organisms and, therefore, ultimately end up at depth rather than re-circulating in surface waters where it could be returned to the atmosphere. Also there are less cellular fragments generated by lysis that could be photo-oxidised by the sun or respired by bacteria. Future experimental and field work should seek to test the results of these models in oceanic or laboratory systems.

\section{Acknowledgements}

CJR is supported by the Research Councils of the United Kingdom. APM is supported by NERC and this work forms part of NOCS contribution to Theme 2 of NERC funded Oceans 2025. The authors thank V. A. A. Jansen (Royal Holloway College, University of London) and J. Truscott (IC London) for discussions of plankton models, and S. Kimmance and S. Archer (Plymouth Marine Laboratory) for useful discussions on phytoplankton viruses. The authors thank the referees for their comments. 


\section{Tables}

\begin{tabular}{|l|l|l|l|}
\hline Parameter & Units & Value & \\
\hline $\mathrm{s}$ & day $^{-1}$ & 0.00648 & Vertical mixing rate \\
\hline $\mathrm{N}_{0}$ & ${\text { mmol } ~^{-3}}^{-3}$ & Variable & Deep NO3 concentration \\
\hline $\mathrm{k}_{\mathrm{N}}$ & ${\text { mmol } \mathrm{N}^{-3}}^{-1}$ & 0.5 & Half-saturation for NO3 uptake \\
\hline$\lambda_{\max }$ & day $^{-1}$ & 0.66 & P maximum growth rate \\
\hline$\mu_{P_{s}}$ & day $^{-1}$ & 0.03 & Natural mortality rate for $\mathrm{P}$ \\
\hline $\mathrm{g}$ & day $^{-1}$ & 2.0 & Maximum grazing rate \\
\hline$\varepsilon$ & $\left(\mathrm{mmol} \mathrm{N} \mathrm{m}^{-3}\right)^{-2}$ day $^{-1}$ & 1.0 & Slope of grazing function \\
\hline$\gamma_{1}$ & & 0.75 & Assimilation efficiency \\
\hline$\gamma_{2}$ & day $^{-1}$ & 0.03 & Excretion rate \\
\hline$\mu_{Z}$ & $\left(\mathrm{mmol} \mathrm{N} \mathrm{m}^{-3}\right)^{-1}$ day $^{-1}$ & 0.15 & Z mortality rate \\
\hline$\mu_{D}$ & day $^{-1}$ & 0.05 & Remineralisation rate \\
\hline$w_{s}$ & m day $^{-1}$ & 5.0 & Sinking rate \\
\hline $\mathrm{h}$ & m $^{-1}$ & 25.0 & Mixed layer depth \\
\hline$\mu_{P_{i}}$ & day $^{-1}$ & 0.16 & Infected P mortality rate \\
\hline$\gamma$ & day $^{-1}$ & 0.16 & Rate of viral production \\
\hline$\mu_{V}$ & day $^{-1}$ & 1.23 & Viral mortality rate \\
\hline
\end{tabular}

Table 1: List of ecosystem and epidemiological parameters used in Model 3. 


\section{Appendix A}

A simple ecological model of a population $P$ subject to logistic growth (growth rate $a$ and carrying capacity $K$ ) and a fatal infectious disease (with transmissibility $\beta$ and with the lifetime of the infected organism set by $\alpha^{-1}$ ) is given by

$$
\begin{gathered}
\frac{d P_{s}}{d t}=a P_{s}\left(1-\frac{P_{s}}{K}\right)-\beta P_{s} P_{i} \\
\frac{d P_{i}}{d t}=\beta P_{s} P_{i}-\alpha P_{i}
\end{gathered}
$$

where the non-infected and infected individuals are partitioned into two compartments $P_{s}$ and $P_{i}$. The model has three steady states $\left(P_{s, 1}{ }^{*}, P_{i, 1}^{*}\right)=(0,0),\left(P_{s, 2}{ }^{*}, P_{i, 2}^{*}\right)=(K, 0)$ and $\left(P_{s, 3}^{*}, P_{i, 3}^{*}\right)=(\alpha / \beta,(a / \beta)(1-\alpha / \beta K))$. As nutrient input, set by $K$, rises, the density of infected individuals, $P_{i}$, increases.

For steady state 3 , the Jacobian is

$$
J=\left(\begin{array}{cc}
\frac{-a \alpha}{K \beta} & -\alpha \\
a\left(1-\frac{\alpha}{K \beta}\right) & 0
\end{array}\right)
$$

From A3, $\operatorname{trace}(J)<0$ for all parameter values.

Likewise, determinant $(J)=a \alpha(1-\alpha / K \beta)=\alpha \beta P_{i, 3}^{*}$ which is $>0$. So steady state 3 is a stable spiral or stable node. 


\section{Appendix B}

Analysis of Model 1.

The ecological component of Model 1 is given by

$$
\begin{gathered}
\frac{d P}{d t}=a P\left(1-\frac{P}{K}\right)-R_{m} Z P \\
\frac{d Z}{d t}=\delta R_{m} Z P-\mu Z
\end{gathered}
$$

and has three steady states $\left(P_{1}^{*}, Z_{1}^{*}\right)=(0,0),\left(P_{2}^{*}, Z_{2}^{*}\right)=(K, 0)$ and $\left(P_{3}^{*}, Z_{3}^{*}\right)=\left(\mu / \delta R_{m},\left(a / R_{m}\right)\left(1-\mu / K \delta R_{m}\right)\right)$.

For steady state 3 , the Jacobian is

$$
J=\left(\begin{array}{cc}
\frac{-a \mu}{K \delta R_{m}} & \frac{-\mu}{\delta} \\
\delta a\left(1-\frac{\mu}{K \delta R_{m}}\right) & 0
\end{array}\right)
$$

From B3, trace $(J)<0$ for all parameter values.

Likewise, determinant $(J)=\mu a\left(1-\mu / K \delta R_{m}\right)=\mu R_{m} Z_{3}^{*}$ which is $>0$. So steady state 3 is a stable spiral or stable node.

As $K$ increases, the steady state 3 fixed point does not change its characteristics because the trace and determinant remain $<0$ and $>0$ respectively. Note also that $\partial P_{3}^{*} / \partial K=0$, indicating that phytoplankton density is independent of nutrient level in the absence of virus. 


\section{Appendix C}

As viral transmissibility increases further above $\left(\delta R_{m} / \mu\right)(a+\alpha)-(a / K)$, the zooplankton population declines and eventually becomes small. In fact, the zooplankton population is eliminated for a sufficiently large $\beta\left(\beta_{c}\right)$. To find this value set equation 6 to zero, i.e.

$$
Z^{*}=\frac{\beta K}{R_{m}}\left(1-\frac{\beta \mu}{\delta R_{m} a}+\frac{\alpha}{a}\right)-\frac{\alpha}{R_{m}}=0
$$

This is satisfied for

$$
\frac{\mu}{\delta R_{m} a} \beta^{2}-\left(\frac{\alpha}{a}+1\right) \beta+\frac{\alpha}{K}=0
$$

which has two real roots, the largest of which is

$$
\beta_{c}=\frac{\delta R_{m} a}{2 \mu}\left(\frac{\alpha}{a}+1\right)+\frac{\delta R_{m} a}{2 \mu}\left(\frac{\alpha^{2}}{a^{2}}+1+2 \frac{\alpha}{a}-4 \frac{\mu \alpha}{2 \delta R_{m} a K}\right)^{1 / 2}
$$

(The other root gives a solution which lies in the region where virus is absent, but for small $K$ there is the possibility that the lower bound in equation 9 might need to be replaced by the small second root of equation $\mathrm{C} 2$ ).

We can show that $\beta_{c}$ is always lower than the upper limit given in equation 9.

Dividing equation $\mathrm{C} 3$ by the upper limit in equation 9 gives $(1-X)^{1 / 2}$ where $X=2 \mu \alpha a / \delta R_{m} K(a+\alpha)^{2}$ which because $X$ is positive means the ratio is always $<1$. 
Alternatively for large $K$ equation $\mathrm{C} 3$ can be approximated by

$$
\beta_{c}=\frac{\delta R_{m}}{\mu}(\alpha+a)-\frac{\alpha}{(1+\alpha / a) K}+\ldots . O\left(K^{-2}\right)
$$

Hence, there is no interval in which $\partial P_{i}^{*} / \partial K>0$ is observable and with zooplankton, phytoplankton and virus co-existing. $\beta_{c}$ is shown by the solid vertical line in Figure 1.

For $\beta>\beta_{c}$ the system consists only of infected and uninfected phytoplankton (but no zooplankton) given by

$$
P_{i}^{*}=\frac{a}{\beta}\left(1-\frac{\alpha}{\beta K}\right)
$$

And

$$
P_{s}^{*}=\frac{\alpha}{\beta}
$$




\section{Appendix D}

Analysis of Model 2.

The ecological component of Model 2 is given by

$$
\begin{gathered}
\frac{d P}{d t}=a P\left(1-\frac{P}{K}\right)-\frac{\lambda Z P}{g+P} \\
\frac{d Z}{d t}=\frac{\delta \lambda Z P}{g+P}-\mu Z
\end{gathered}
$$

has three steady states $\left(P_{1}^{*}, Z_{1}^{*}\right)=(0,0),\left(P_{2}^{*}, Z_{2}^{*}\right)=(K, 0)$ and $\left(P_{3}^{*}, Z_{3}^{*}\right)=(\mu g /(\delta \lambda-\mu),(a g / \lambda)(1+\mu /(\delta \lambda-\mu))(1-\mu g / K(\delta \lambda-\mu)))$.

The Jacobian for Model 2 is given by

$$
J=\left(\begin{array}{cc}
a-\frac{2 a P^{*}}{K}-\frac{g \lambda Z^{*}}{\left(g+P^{*}\right)^{2}} & -\frac{\lambda P^{*}}{\left(g+P^{*}\right)} \\
\frac{\delta \lambda Z^{*} g}{\left(g+P^{*}\right)^{2}} & \frac{\delta \lambda P^{*}}{\left(g+P^{*}\right)}-\mu
\end{array}\right)
$$

At $\left(P_{3}^{*}, Z_{3}^{*}\right)$ the term in the lower right of the Jacobian is zero. Therefore,

Det $J>0$ because all the terms in the top right and bottom left entries are always positive at steady state 3 . Consequently, the trace of the Jacobian at this steady state is

$$
\operatorname{Tr} J=a-\frac{2 a P_{3}^{*}}{K}-\frac{g \lambda Z_{3}^{*}}{\left(g+P_{3}^{*}\right)^{2}}
$$

For stable fixed point behaviour $\operatorname{Tr} J<0$, and for this to be the case 


$$
1<\frac{2 P_{3}^{*}}{K}+\frac{\left(1-P_{3}^{*} / K\right)}{\left(1+P_{3}^{*} / g\right)}
$$

The second term in equation D5 is typically small, so for stable fixed points at stationary state 3 requires

$$
1<\sim \frac{2 \mu g}{K(\delta \lambda-\mu)}
$$




\section{Appendix E}

From equation 16 it is possible to find for Model 2 the critical value of $\beta\left(\beta_{c}\right)$ above which the zooplankton population is eliminated. Repeating the analysis of Appendix $\mathrm{C}$ with this equation, we find

$$
\beta_{c}=\frac{(\delta \lambda-\mu)(\alpha+a)}{\mu g}-\frac{\alpha}{K(1+\alpha / a)}+. . O\left(K^{-2}\right)
$$

Therefore, for all viruses that stably co-exist with the phytoplankton and zooplankton, any increase in nutrient leads to declining prevalence of infected phytoplankton. $\beta_{c}$ is shown by the solid vertical line in Figure 3. 


\section{Figure Captions}

Figure 1: The equilibrium value of $P_{i}^{*}(\mu g N / l)$ is plotted as a function of viral transmissibility $\beta(\mu g N / l)^{-1}$ day $^{-1}$ for Model 1 . The model parameter values used in this plot are illustrative and more specifically take the following values $a=0.3$ day $^{-1}, K=108 \mu g N / l, R m=0.05(\mu g N / l)^{-1}$ day $^{-1}, \delta=0.05, \mu=0.012$ day $^{-1}, \alpha=0.16$ day $^{-1}$ . For insufficiently transmissible virus (to the left of the l.h.s. vertical dashed line) the virus is unable to establish itself in the phytoplankton population, denoted by $P$. In the region bounded by the l.h.s. vertical dashed line and the vertical solid line is where nutrient enrichment will reduce the prevalence of infected phytoplankton. Note that Model 1 biomass units are $(\mu g N / l)$ for consistency with Truscott and Brindley (1994) and Rhodes et al. (2008).

Figure 2a: The infected and uninfected phytoplankton biomass $(\mu g N / l)$ is plotted one above the other for Model 1 to show how the proportions change as nutrient increases. Infected phytoplankton is eliminated for a nutrient level $>K_{c}$. For higher nutrient levels, the system is purely ecological with phytoplankton,$P$, and zooplankton, $Z$, only. Zooplankton biomass $(\mu g N / l)$ is represented by the black dots. In this plot the parameters of the model are the same as in Figure 1, but with a fixed $\beta=0.094(\mu g N / l)^{-1}$ day $^{-1}$.

Figure 2b: Plot showing the variation of infected phytoplankton biomass $(\mu g N / l)$ as a function of nutrient input $K$ and disease transmissibility $\beta$. The parameter region shown is $190<K<130(\mu g N / l)$ and $0.0925<\beta<0.0955(\mu g N / l)^{-1}$ day $^{-1}$.

Figure 3: Repeat of Figure 1 using Model 2 with the following parameters: $a=0.3$ day $^{-1}, K=108 \mu g N / l, \lambda=0.3$ day $^{-1}, g=15 \mu g N / l, \delta=0.05, \mu=0.012$ day $^{-1}, \alpha=0.16$ day $^{-1}$ 
Figure 4a: Repeat of Figure 2 using Model 2 with a fixed $\beta=0.006(\mu g N / l)^{-1} \mathrm{day}^{-1}$. The other model parameters are the same as used in Figure 3.

Figure 4b: Plot showing the variation of infected phytoplankton biomass $(\mu g N / l)$ as a function of nutrient input $K$ and disease transmissibility $\beta$. The parameter region shown is $190<K<130(\mu g N / l)$ and $0.0045<\beta<0.0075(\mu g N / l)^{-1}$ day $^{-1}$.

Figure 5a: The uninfected and infected biomass $\left(\mathrm{mmol} \mathrm{N} \mathrm{m}^{-3}\right)$ as a function of ambient nutrient level $\left(\mathrm{mmol} \mathrm{N} \mathrm{m}^{-3}\right)$ for Model 3. Also shown is the zooplankton biomass (black dots). Parameter values can be found in Table 1. Note that Model 3 biomass units are mmol $\mathrm{N} \mathrm{m}^{-3}$ for consistency with Rhodes et al. (2008).

Figure 5b: The uninfected and infected plankton biomass $\left(\mathrm{mmol} \mathrm{N} \mathrm{m}^{-3}\right)$ proportions as a function of the total phytoplankton biomass. Parameter values can be found in Table 1.

Figure 6a: The uninfected and infected phytoplankton biomass $\left(\mathrm{mmol} \mathrm{N} \mathrm{m}^{-3}\right)$ as a function of ambient nutrient level $\left(\mathrm{mmol} \mathrm{N} \mathrm{m}^{-3}\right)$; Model 3 for $\beta=4.5 \mathrm{mmol} \mathrm{N} \mathrm{m}^{-3}$ day $^{-}$ ${ }^{1}$. Other parameter values can be found in Table 1.

Figure 6b: same calculation as Figure $6 \mathrm{a}$, for $\beta=5.0 \mathrm{mmol} \mathrm{N} \mathrm{m}^{-3} \mathrm{day}^{-1}$. Other parameter values can be found in Table 1 .

Figure 6c: same calculation as Figure 6a, for $\beta=5.5 \mathrm{mmol} \mathrm{N} \mathrm{m}^{-3}$ day $^{-1}$. Other parameter values can be found in Table 1 .

Figure 6d: same calculation as Figure 6a, for $\beta=6.0 \mathrm{mmol} \mathrm{N} \mathrm{m}^{-3} \mathrm{day}^{-1}$. Other parameter values can be found in Table 1 .

For each plot the abundance of the infected and uninfected phytoplankton are stacked one on top of the other, so the total height of the bars represents the total phytoplankton abundance $\left(P_{s}+P_{i}\right)$. Zooplankton population density increases monotonically with increasing nutrient enrichment, as in Figure 5a.

Figure 7: Time series of nitrate levels $\left(\mathrm{mmol} \mathrm{N} \mathrm{m}^{-3}\right)$ and viral-induced mortality $\left(\right.$ days $\left.^{-1}\right)$ in the phytoplankton Phaeocystis globosa. Data is taken from Baudoux et al. 
(2006), using seawater samples collected from the southern North Sea from March to May. At high nitrate levels viral-induced mortality of phytoplankton is minimal, whereas at low nitrate levels viral-induced mortality is much higher. 


\section{References}

Anderson, R. M., May, R. M. 1986. The invasion, persistence and spread of infectious diseases within animal and plant communities. Phil. Trans. R. Soc. Lond. B314, 533570.

Arrigo, K. R. 2005. Marine micro-organisms and global nutrient cycles. Nature 437, 349-355.

Baudoux, A., Noordeloos, A. A. M.,Veldhuis, M. J. W., Brussard, C. P. D., 2006.Virally induced mortality of Phaeocystis globosa during two spring blooms in temperate coastal waters. Aqua. Microb. Ecol. 44, 207-217.

Begon, M., Townsend, C. A., Harper, J. L. 2005. Ecology: From individuals to ecosystems. Wiley Blackwell, Oxford.

Beltrami, E., Carroll T. O., 1994. Modelling the role of viral disease in recurrent phytoplankton blooms. J. Math. Biol. 32, 857-863.

Bergh, O., Borsheim, K. Y., Bratbak, G., Heldal, M., 1989. High abundance of viruses found in aquatic environments. Nature 340, 476-468.

Bratbak, G., Heldal, M., Norland, S., Thingstad, T. F., 1990. Viruses as partners in spring bloom and microbial trophodynamics. Appl. Environ. Microbiol. 56, 14001405 .

Bratbak, G., Egge, J. K., Heldal, M., 1993. Viral mortality of the marine alga Emiliania huxleyi (Haptophyceae) and termination of algal blooms. Mar. Ecol. Prog. Ser. 93, 39-48. 
Brussard, C. P. D., Kuipers, B., Veldhuis, M. J. W., 2005. A mesocosm study of Phaeocystis globosa population dynamics I. Regulatory role of viruses in bloom control. Harm. Alg. 4, 859-874.

Chisholm, S. W., 2000. Stirring times in the Southern Ocean. Nature, 407, 685-687.

Chisholm, S. W, Falkowski, P. G., Cullen, J. J., 2001. Dis-Crediting ocean fertilization. Science, 249, 309-310.

Chattopadhyay, J., Arino, O., 1999. A predator-prey model with disease in the prey. Nonlin. Anal., 36, 747-766.

Chattopadhyay, J., Pal, S., 2002. Viral infection on phytoplankton-zooplankton system: a mathematical model. Ecol. Mod., 151, 15-28.

Chattopadhyay, J., Sarkar, R. R., Pal., S. 2003. Dynamics of nutrient phytoplankton interaction in the presence of viral infection. Biosys. 68, 5-17.

Edwards, A. M., Brindley, J., 1999. Zooplankton mortality and the dynamical behaviour of plankton population models. Bull. Math. Biol. 61, 303-339.

Evans, C., Archer, S. D., Jacquet, S., Wilson, W. H., 2003. Direct estimates of the contribution of viral lysis and microzooplankton grazing to the decline of a Micromonas spp. population. Aquat. Microb. Ecol. 30, 207-219.

Fuhrman, J. A., 1999. Marine viruses and their biogeochemical and ecological effects. Nature 399, 541-548.

Greenhalgh, D., Haque, M. 2007. A predator-prey model with disease in the prey species only. Math. Meth. Appl. Sci., 30, 911-929.

Grenfell, B. T., Dobson A. P. (Eds) 1995. Ecology of infectious diseases in natural populations. Publications of the Newton Institute, Cambridge University Press, Cambridge. 
Hilker, F. M., Malchow, H. 2006. Strange periodic attractors in a prey-predator system with infected prey. Math. Pop. Stud., 13, 119-134.

Holling, C. S. 1959. Some characteristics of simple types of predation and parasitism. Can. Ent., 91, 385-398.

Hudson, P., Rizzoli, A., Grenfell, B., Heesterbeek, H., Dobson, A., 2002. The Ecology of Wildlife Diseases. Oxford University Press, Oxford.

Kaiser, M. J., Attrill, M. J., Jennings, S., Thomas, D. N., Barnes, D. K. A., Brierley, A. S., Polunin, N. V. C., Raffaelli, D. G., Williams, P. J. le B. 2005. Marine Ecology: Processes, Systems and Impacts, Oxford University Press, Oxford.

Lampitt, R.S., Achterberg, E.P., Anderson, T.R., Hughes, J.A., Iglesias-Rodriguez, M.D., Kelly-Gerreyn, B.A., Lucas, M., Popova, E.E., Sanders, R., Shepherd, J.G., Smythe-Wright, D. and Yool, A. (2008) Ocean fertilisation: a potential means of geoengineering?_Philosophical Transactions of the Royal Society A, 366, (1882), 39193945.

Lovelock, J. E. and Rapley, C. G. 2007 Ocean pipes could help the earth cure itself. Nature, 449, 403.

Malchow, H., Hilker, F. M., Petrovskii, S. V., Brauer, K. 2004. Oscillations and waves in a virally infected plankton system Part I: The lysogenic stage. Ecol. Compl., $1,211-223$.

Malchow H., Hilker F. M., Sarkar R. R., Brauer K., 2005. Spatiotemporal patterns in an excitable plankton system with lysogenic viral infection. Math and Comp. Modelling, 42, 1035-1048.

Mann, N. H., Cook, A., Millard, A., Bailey, S., Clokie, M. 2003. Bacterial photosynthesis genes in a virus. Nature, 424,741 
Martin, A. P., Richards, K. J., Bracco, A., Provenzale, A., 2002. Patchy productivity in the open ocean. Glob Biogeo Cycl. 16,2, 9-1 - 9-9.

Oschlies, A., Garçon V., 1999. An eddy-permitting coupled physical-biological model of the North Atlantic 1: sensitivity to advection numerics and mixed layer physics. Glob. Biogeo. Cyc. 13, 135-160.

Rhodes, C. J., Truscott, J. E., Martin, A. P. 2008. Viral infection as a regulator of oceanic phytoplankton populations. J. Mar. Sys., 74, 216-226.

Rosenzweig, M., 1971. Paradox of Enrichment: destabilisation of exploitation ecosystems in ecological time. Science, 171, 385-387.

Rosenzweig, M., 1972. Reply to "Stability of enriched aquatic ecosystems". Science, $175,564-565$.

Sabine, C. L., Feely, R. A., Gruber, N., Key, R. M., Lee, K., Bullister, J. L., Wanninkhof, R., Wong, C. S., Wallace, D. W. R., Tilbrook, B., Millero, F. J., Peng, T. H., Kozyr, A., Ono, T., Rios, A. F., 2002. The oceanic sink for anthropogenic $\mathrm{CO}_{2}$. Science, 305, 367-371.

Siekmann, I., Malchow, H., and Venturino, E. 2008. An extension of the BerettaKuang model of viral diseases. Math. Biosci. Eng. 5, 549-565.

Singh, B. K., Chattopadhyay, J., Sinha, S., 2004. The role of virus infection in simple phytoplankton zooplankton system. J. Theo. Biol., 231, 153-166.

Steele, J. H, Henderson, E. W., 1992. The role of predation in plankton models. J. Plankton. Res. 14, 676-691.

Suttle, C. A, Chan, A. M., Cottrell, M. T., 1990. Infection of phytoplankton by viruses and reduction of primary production. Nature, 387, 467-469.

Suttle, C. A., 2005. Viruses in the sea. Nature, 437, 356-361. 
Thyrhaug R., Larsen, A., Thingstad, T. F., Bratbak, G., 2003. Stable co-existence in marine algal host-virus systems. Mar. Ecol. Prog. Ser. 254, 27-35.

Truscott, J. E., Brindley J., 1994. Ocean plankton populations as excitable media. Bull. Math. Biol. 56, 981-998.

Xiao, Y., Chen, L., 2001. Modelling and analysis of a predator-prey model with disease in the prey. Math. Biosc., 171, 59-82. 


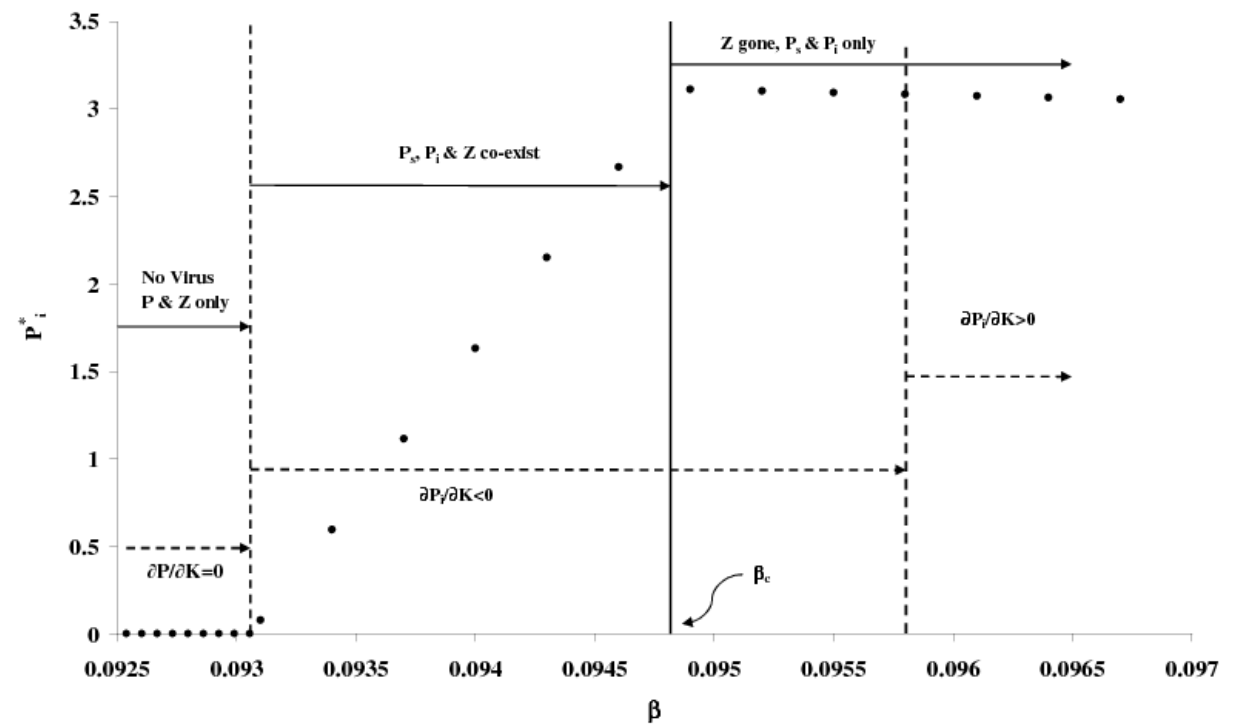




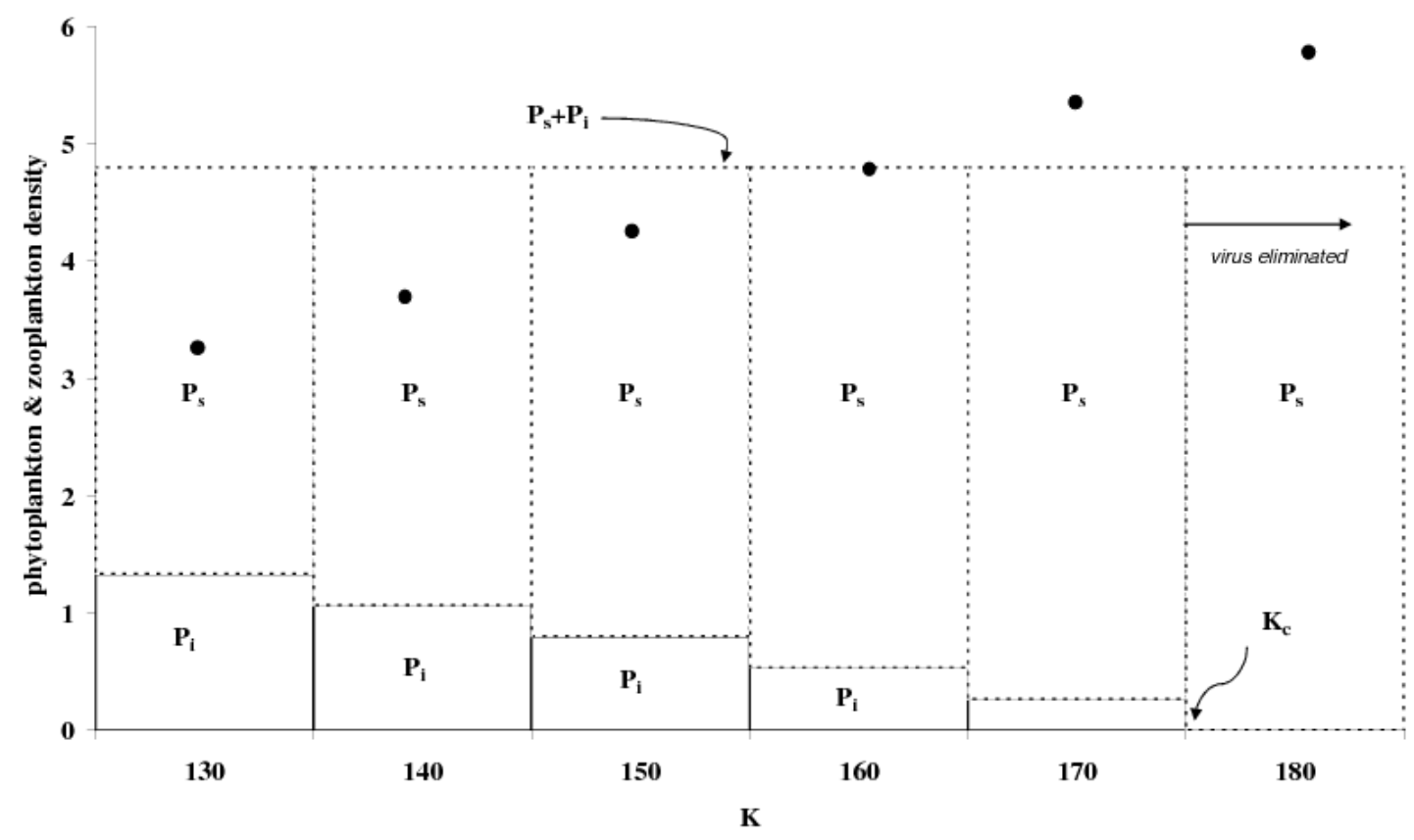




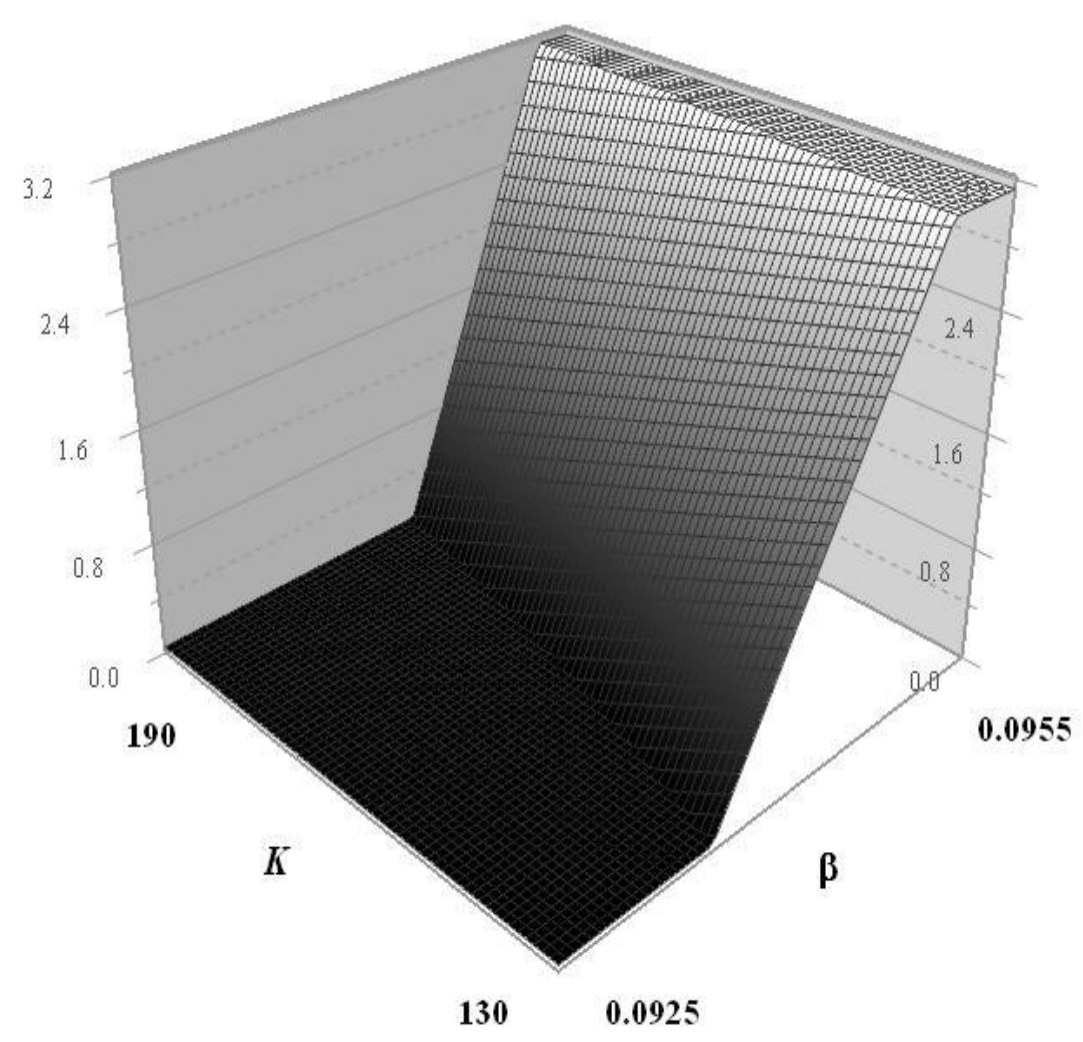




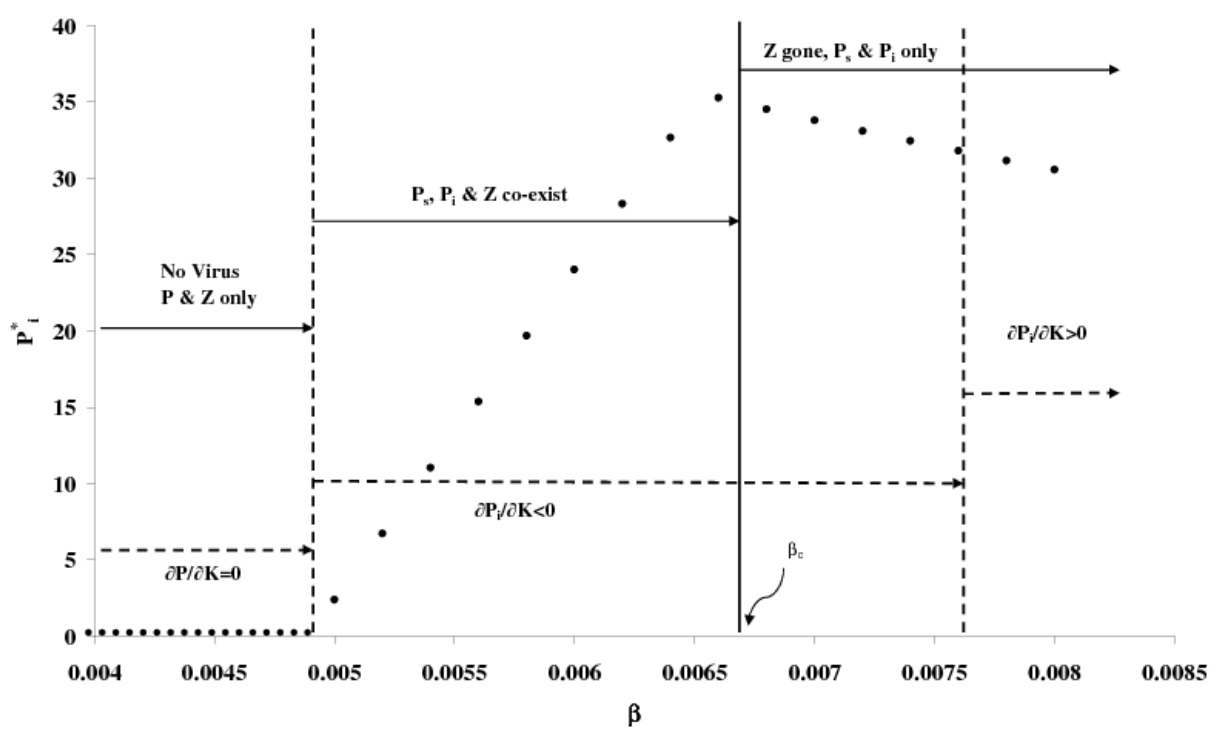




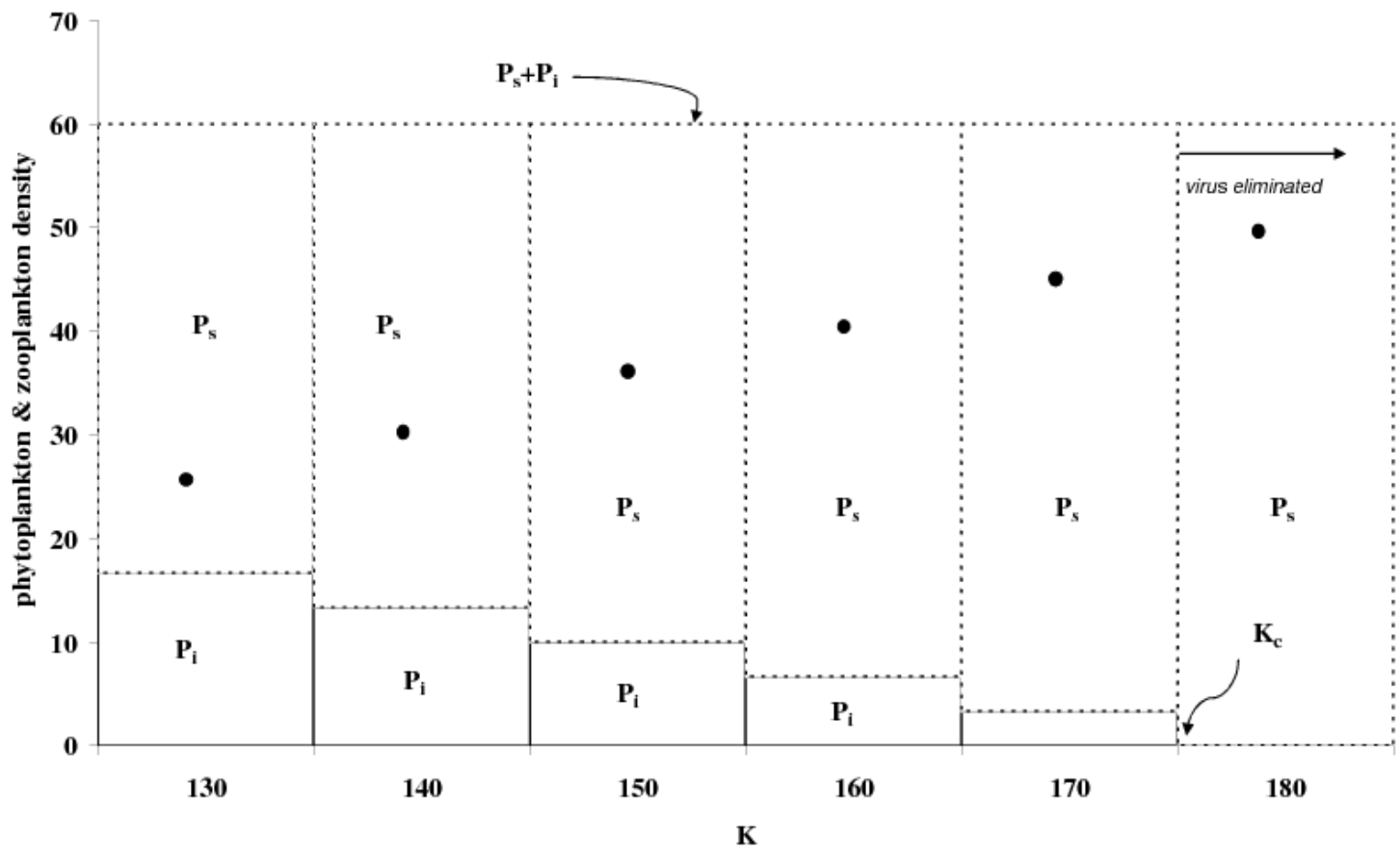




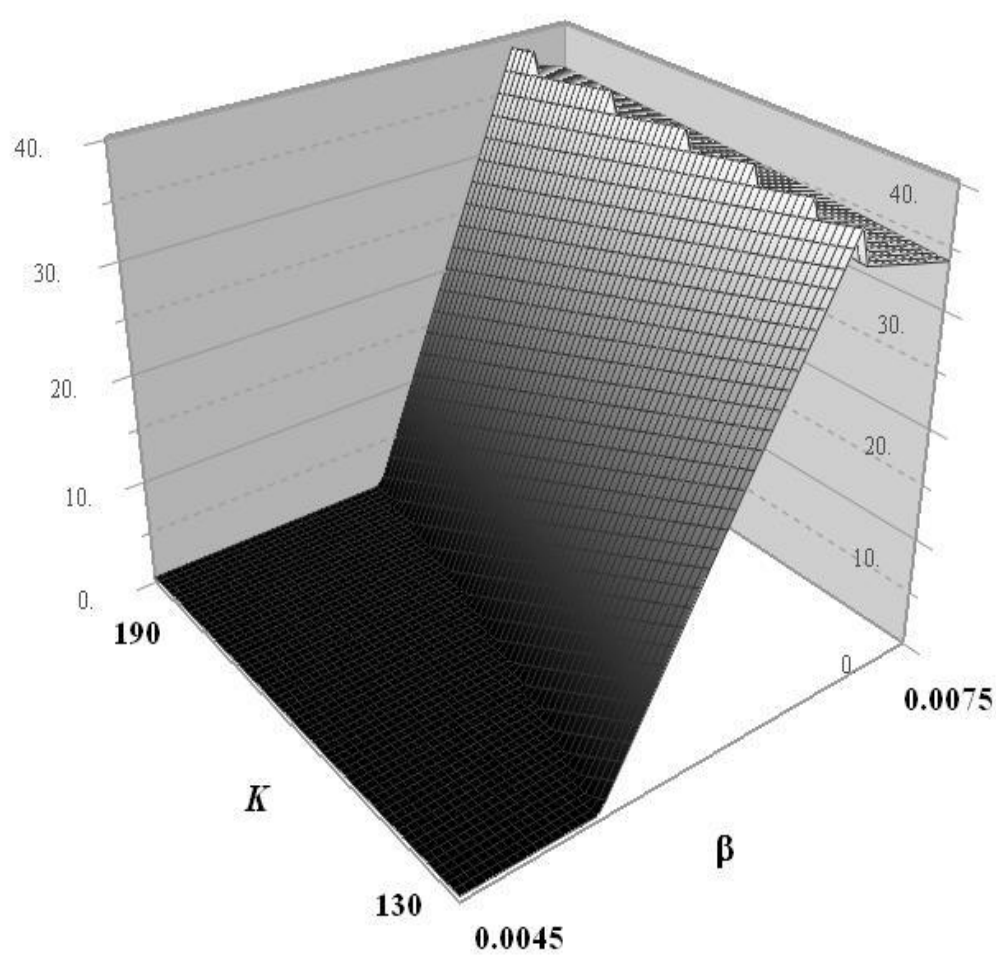




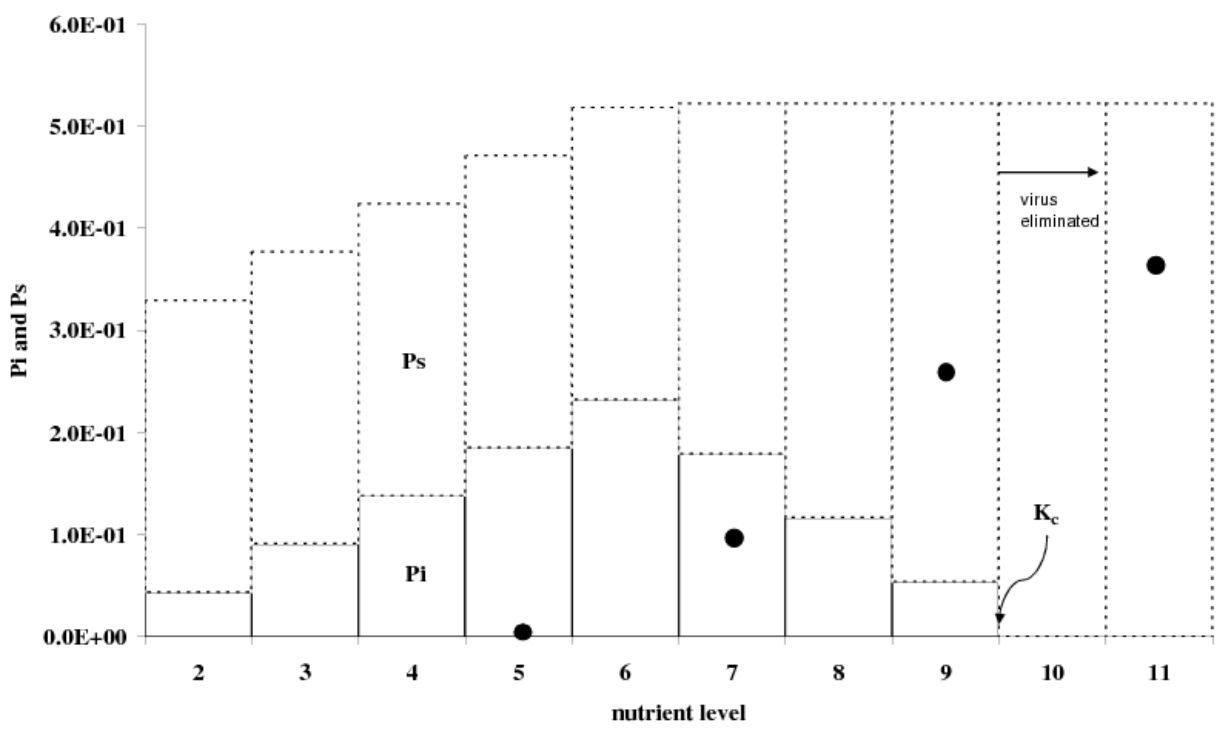




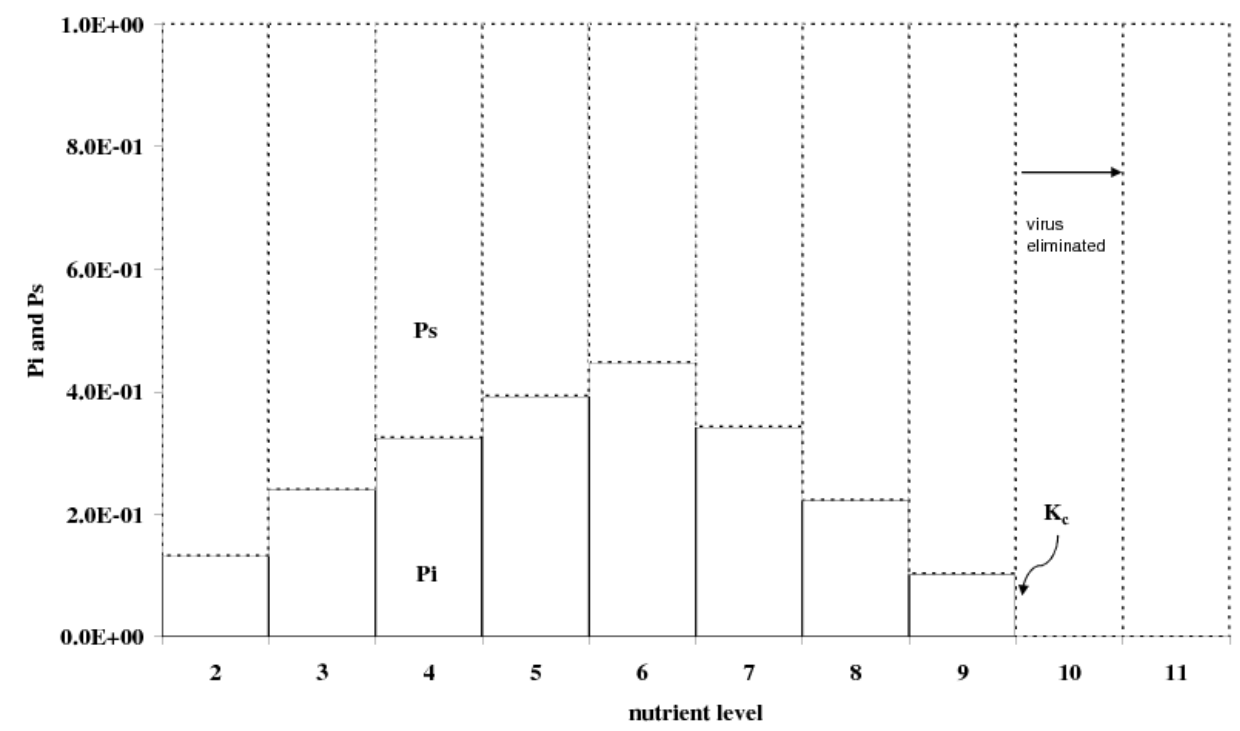




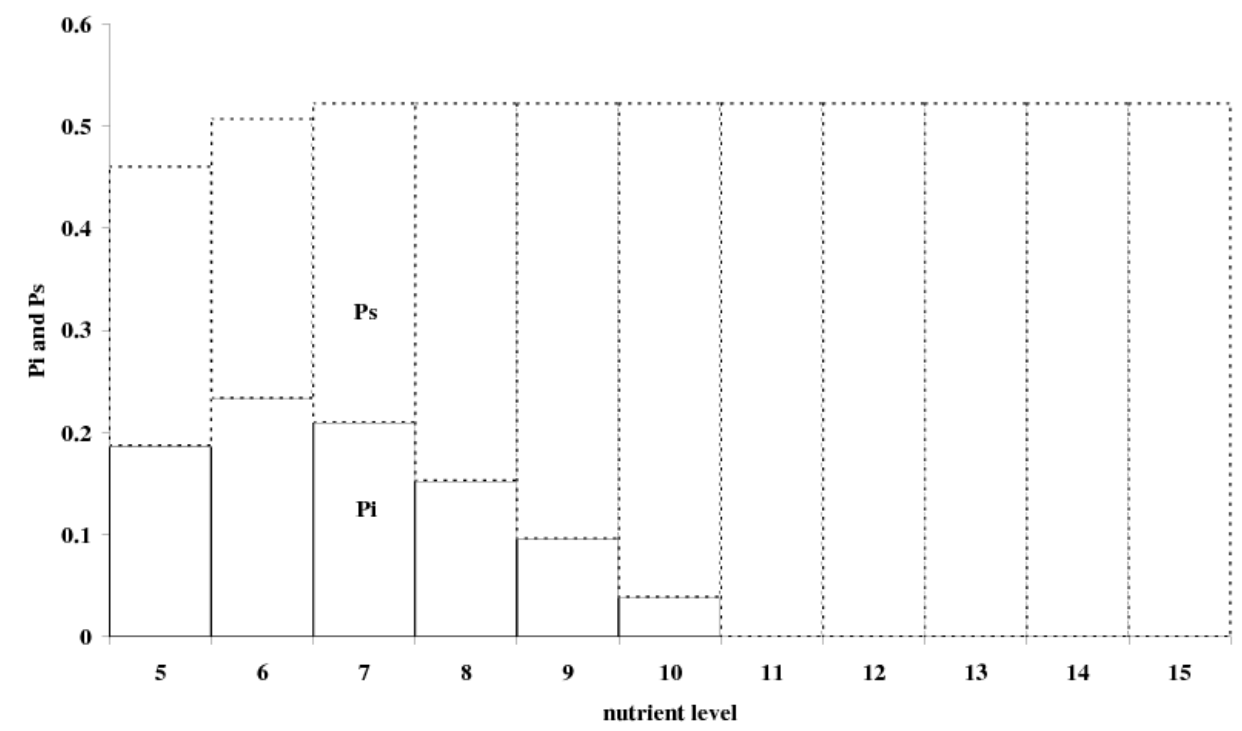




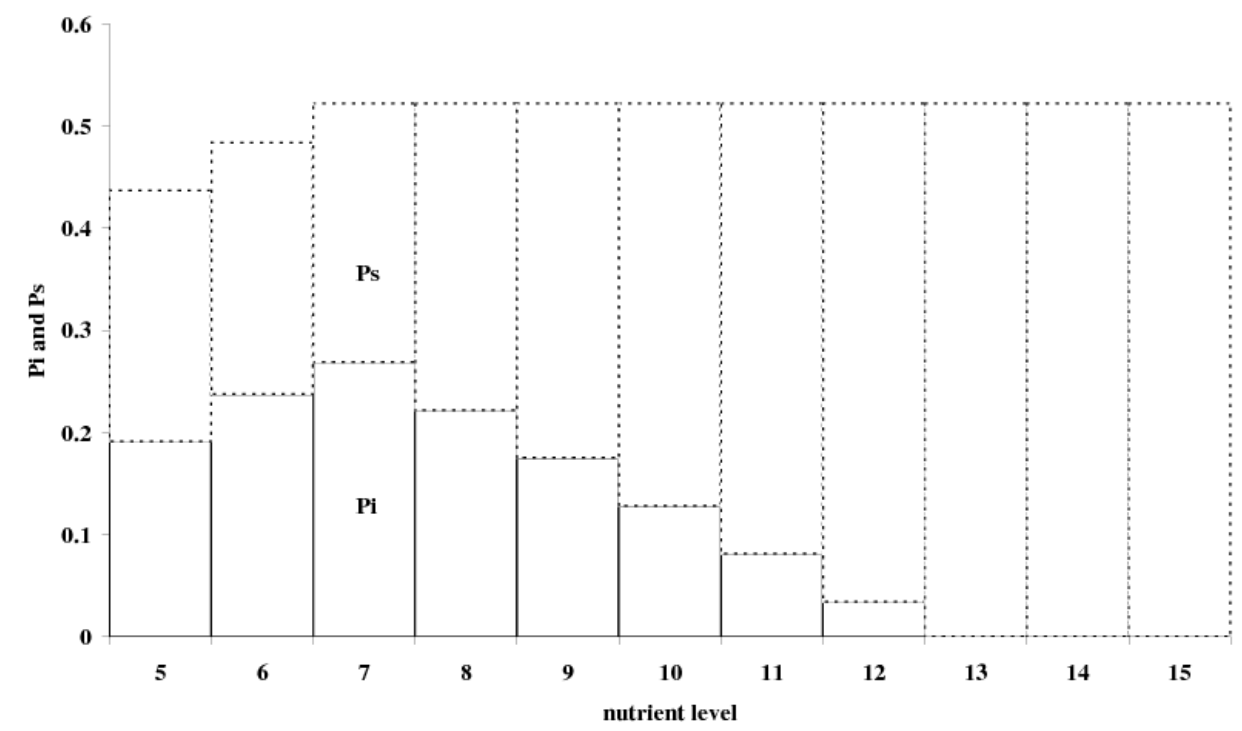




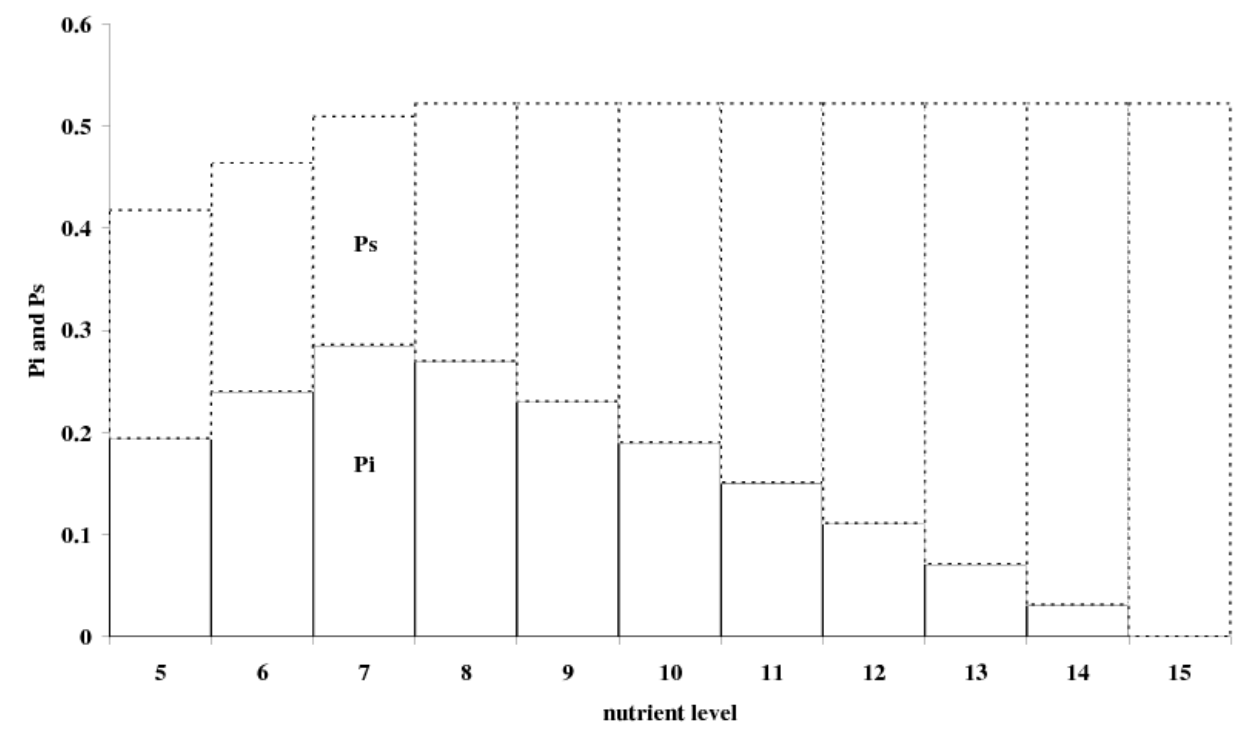




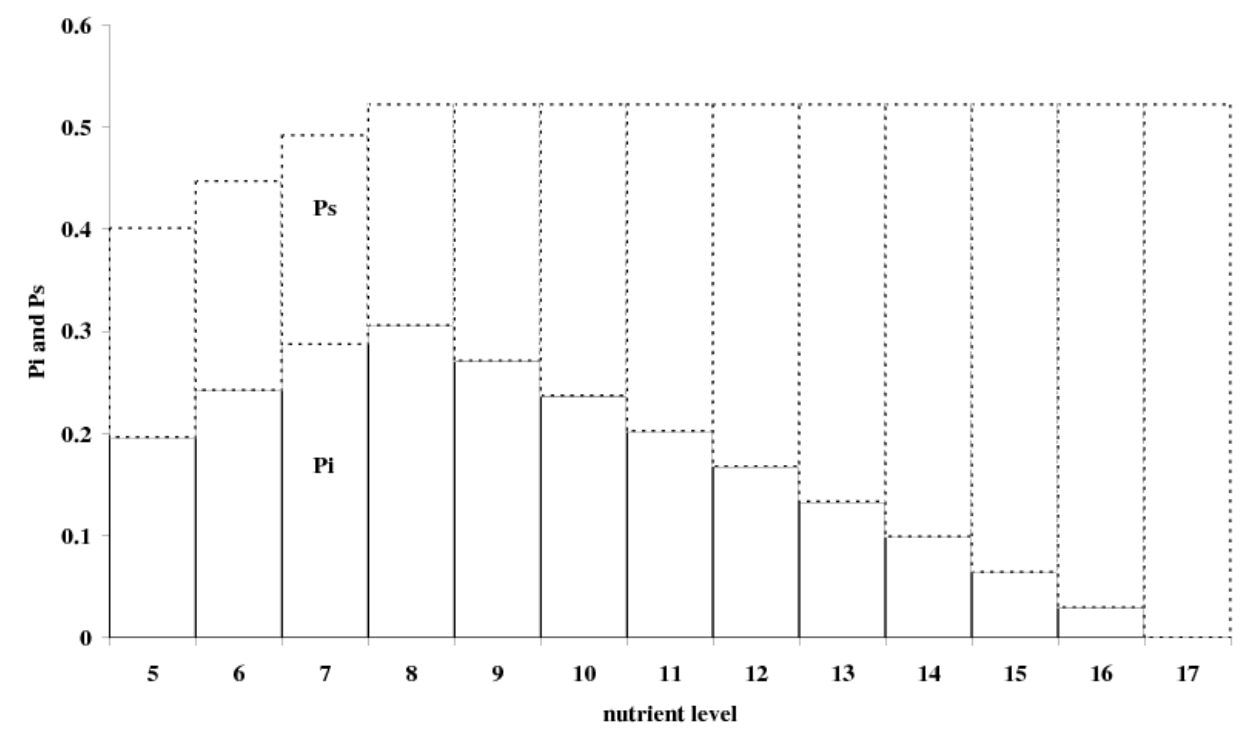




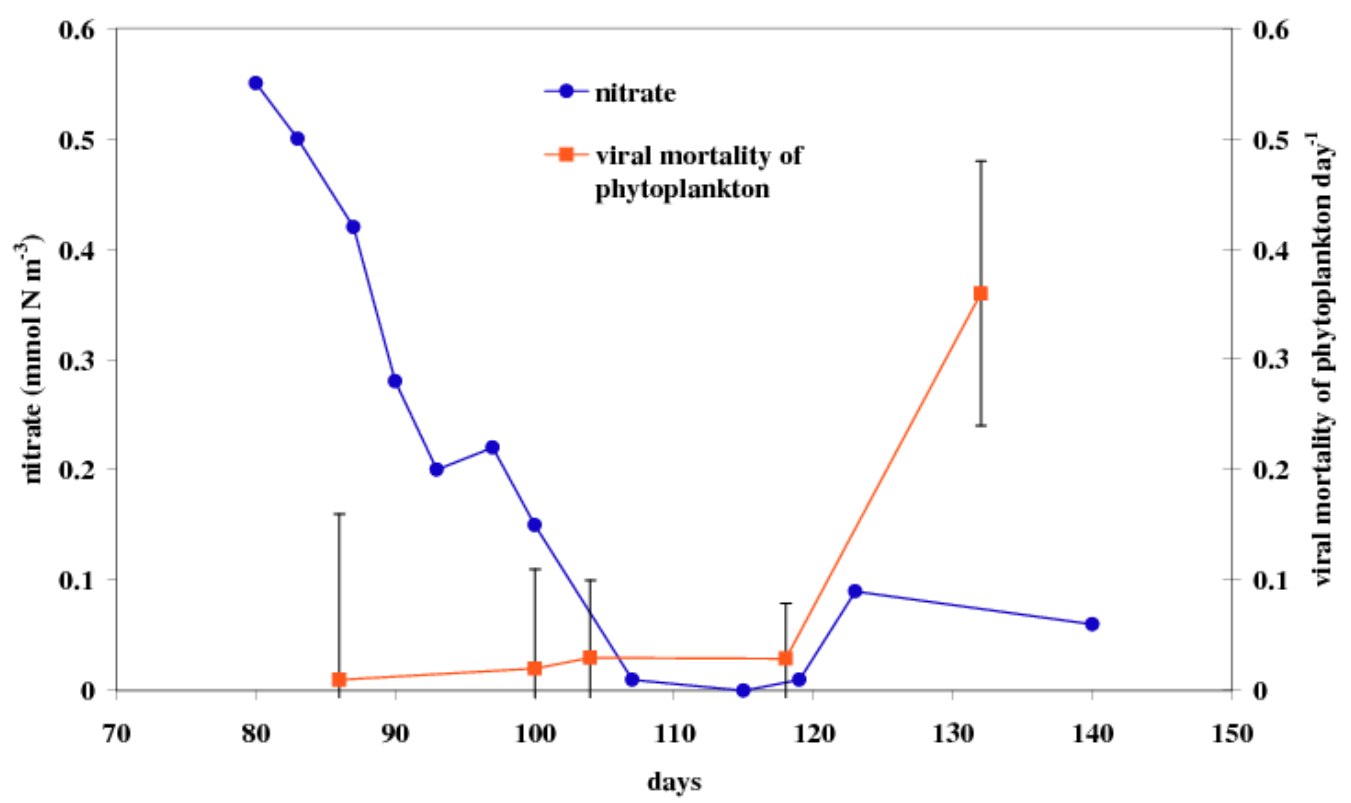

